\title{
Dynamic problems for metamaterials: review of existing models and ideas for further research
}

\author{
Dionisio Del Vescovo ${ }^{1,2}$ and Ivan Giorgio ${ }^{1,2}$
}

16th February 2014

\author{
${ }^{1}$ Dipartimento di Ingegneria Meccanica e Aerospaziale, Facoltà di Ingegneria \\ Civile e Industriale, Università di Roma La Sapienza, Via Eudossiana 18, \\ 00184, Roma, Italia \\ ${ }^{2}$ International Research Centre for the Mathematics and Mechanics of \\ Complex Systems "M\&MoCS"
}

\begin{abstract}
Metamaterials are materials especially engineered to have a peculiar physical behaviour, to be exploited for some well-specified technological application. In this context we focus on the conception of general microstructured continua, with particular attention to piezoelectromechanical structures, having a strong coupling between macroscopic motion and some internal degrees of freedom, which may be electric or, more generally, related to some micro-motion. An interesting class of problems in this context regards the design of wave-guides aimed to control wave propagation.

The description of the state of the art is followed by some hints addressed to describe some possible research developments and in particular to design optimal design techniques for bone reconstruction or systems which may block wave propagation in some frequency ranges, in both linear and non-linear fields.
\end{abstract}

Keywords: Metamaterials, micro-structured media, micropolar continuum, micromorphic continuum, variational principles.

\section{Introduction}

At the very beginning of this paper the authors clarify that the use of the word metamaterial is intended as a synonym of microstructured continuum. One could question this choice, but, according to (Eringen, 2001), whenever a continuum model is applicable then a suitable microstructural set of kinematical descriptors can be determined which effectively describe the considered complex system. Of course when wavelengths are small enough to interact with the discrete microstructure then continuum models will not be applicable. We limit ourselves, therefore, to deal with wave phenomena not influenced by the very fine length micro-scale. 
Metamaterials are materials which are designed to have exotic behaviour: the concept has been first conceived for optical devices. Therefore, very often one talks about mechanical metamaterials, when the exotic behaviour is limited to mechanical effects, as e.g. very negative Poisson effects.

This paper is based on a really simple idea: construct a bridge between two different cultural environments which address the same relevant problems. Metamaterials are studied and conceived by physicists to tackle problems and applications not yet considered in engineering sciences; at the same time the community of continuum mechanics nearly completely ignores what physicists devise and develop in the same field. This review intends to fill a gap in order to stimulate a parallel development of the these theories and to near not communicating scientific groups.

The mathematical formalism chosen thereinafter is that preferred by physicist, like Landau type variational principles, and the treated subjects are chosen, in the opinion of the authors, from those considered nowadays more important by applied mechanicians.

The capability of continuum theories to describe the time evolution and the deformation of the micro-structure of complex mechanical systems was recognised in the very first formulations of continuum mechanics, as in the pioneering work by Piola (Piola, 1846). He was lead by stringent physical considerations to introduce higher gradients of displacement field, as necessary independent variables, in the constitutive equation for the deformation energy of continuous media. For a more modern interpretation of this subject refer to (Mindlin, 1964, 1965; Mindlin, Eshel, 1968; Toupin, 1962, 1964; Eringen, 1999, 2001, 2002; dell'Isola, Seppecher, 1995; dell'Isola, Sciarra, Vidoli, 2009; Sciarra, dell'Isola, Coussy, 2007; Auffray, Bouchet, Brechet, 2009).

However in a similar period, while Piola was producing his papers, Cauchy and Poisson obtained a description, with a very elegant and effective format, for continuum mechanics in which:

i) the displacement from a reference configuration is the only kinematic descriptor;

ii) the crucial conceptual tool is Cauchy stress which is constitutively related only to the first gradient of displacement;

iii) the crucial postulates are those concerning balance of mass, linear and angular momentum and, when necessary, energy.

The Cauchy-Poisson format is usually very effective to describe the mechanical behaviour of a very wide class of natural and also artificial materials. Nevertheless, when considering materials with very well-organised microstructures, subjected to particular loads and/or boundary conditions, Cauchy's continuum theory may not be accurate. This is the case of some engineered materials having high contrast of material properties (Alibert, Seppecher, dell'Isola, 2003; dell'Isola, Rosa, Woźniak, 1997, 1998; Ferretti et al., 2013; Pideri, Seppecher, 1997; Camar-Eddine, Seppecher, 2002, 2003) or for some natural materials with highly heterogeneous hierarchical microstructures (Buechner, Lakes, 2003). In all these cases, the introduction of more sophisticated models is mandatory in order to satisfactory catch the features of the mechanical behaviour of such complex materials.

Many and unexpected effects on wave propagation can be mathematically predicted and described in the study of continua with micro-structures (Manktelow, Leamy, Ruzzene, 2013a,b; Narisetti, Ruzzene, Leamy, 2011, 2012; Placidi et 
al., 2013; Rosi, Giorgio, Eremeyev, 2013; Rosi, Madeo, Guyader, 2013; dell'Isola, Madeo, Placidi, 2012; Eremeyev, 2005; Altenbach et al., 2010).

The intent of this review is to indicate the importance of the aforementioned effects and to direct the investigation towards those among them having engineering relevance.

We want to underline that some mathematical results, as those in Ghiba et al. (2013) and Neff et al. (2013), together with the analysis developed in the framework of continuum mechanics (dell'Isola, Madeo, Placidi, 2012; Placidi et al., 2013), can produce interesting hints useful to investigate on exotic effects, i.e. unconventional dynamic behaviours, which can be exploited in engineering applications. For instance, the singular model studied in Ghiba et al. (2013) attracted the attention of the authors because of the difficulties to define some technical upper bounds for the solutions of formulated partial differential equations (PDEs). However the singularity pointed out by the mathematical difficulties is an indication of some peculiarities of this case of micro-morphic, Mindlin-type, micro-structured continua. These peculiarities, afterwards studied and not yet completely described in Neff et al. (2013), promise interesting and probably important applications.

In Mindlin (1964) Mindlin excludes from his analysis the metamaterials considered in Ghiba et al. (2013) and Neff et al. (2013) since their deformation energy is not definite positive as a function of the defined deformation measures: with this meaning the word singular used in a previous sentence should be interpreted.

The challenge consist in using mathematics, continuum modelling, numerical simulations techniques and experiments to determine those material properties which may play a significant role in future technological advances.

In the section dealing with the research perspectives, possible developments are imagined from an applicative point of view.

The literature about metamaterials is enormous and in this paper only some instances are cited.

\section{A Matter of Principle}

We assume that every physical phenomenon can be described by means of a maybe modified or improved version of the Principle of Least Action (Hellinger, 1913). The whole mathematical content of the present work actually is reduced to a general formulation of this Principle and on a series of examples, in which particular Lagrangian functions and, possibly, particular HamiltonRayleigh dissipation functionals are specified to deals with a particular class of phenomena.

\subsection{The Principle of Least Action}

As discussed e.g. in dell'Isola, Placidi (2012), to formulate a mathematical model following the spirit of Lagrange one has to:

1. single out the kinematical descriptors which describe the state of the physical system;

2. choose the set of admissible motions for the system under description; 
3. exert the physical intuition to find the right Action Functional whose minima are exactly the searched motions.

A configuration is the mathematical object used to model the state of the system: the set of possible configurations will be denoted by $C$. The only possible way for labelling a configuration is to find the values of the kinematical descriptors corresponding to it.

The motion is the mathematical model describing the evolution of the system: it is a $C$-valued function defined on time interval $\left(t_{0}, t_{f}\right)$; the set of all admissible motions will be denoted by $M$. Of course one has to assume some regularity properties for the motions in $M$. For instance: to exclude impulsive motions, the regularity hypothesis requires that the second time derivative of $C$-valued function exists.

The action is a real-valued function, defined on $M$ : for traditional reasons and also to avoid to repeat too often the word function, the action is said to be a functional: that is a function defined in a set of functions.

Finally, to use the Principle of Least Action sometimes it is useful to:

1. find the Euler-Lagrange conditions which are consequence of the postulated Least Action Principle;

2. interpret these conditions on a physical ground.

In epochs in which the only method for getting some previsions about the behaviour of a physical system was to find a more or less explicit solution of some system of differential equations, the determination of Euler-Lagrange minimality conditions supplied the fundamental calculation tool for the applied scientists. This is not the case nowadays considering the systematic use of computers in science. It has become progressively more important to determine the numerical integration scheme to adopt in order to predict the model behaviuor, in terms of the postulated Action Functional: therefore the role of Euler-Lagrange equations has become less important. However, the presented integration by parts procedure remains important for determining the boundary conditions.

\subsection{Lagrangian Action Functionals: technical details}

In our presentation we follow more or less closely Landau, Lifshitz (1975, 1976, 1977).

Let $\Psi_{\sigma}\left(x_{\mu}\right)$ be any set of $n$ tensor fields defined on $\mathbb{R}^{m}, \sigma$ being a multi-index and $\mu=1,2, \ldots, m$. We do not want to limit ourselves to three-dimensional or four-dimensional, including time, spaces for defining the fields $\Psi_{\sigma}$. So $e . g$., when dealing with shells we need bi-dimensional domains for $\Psi_{\sigma}$ and when dealing with beams we need one-dimensional domains.

If we define the Lagrangian density as:

$$
\mathfrak{L}\left(x_{\mu}, \Psi_{\sigma}, \frac{\partial \Psi_{\sigma}}{\partial x_{\mu}}\right)
$$

then we can introduce the Action Functional as

$$
\mathfrak{A}=\int_{T} \mathfrak{L}\left(x_{\mu}, \Psi_{\sigma}, \frac{\partial \Psi_{\sigma}}{\partial x_{\mu}}\right)
$$


where $T$ is a hyper-volume in the $m$-dimensional space determined by the $x_{\mu}$.

We remark that this is only a possible choice of Action Functional: however it seems to be general enough to formulate all the models which were conceived up to now.

\subsection{Variation of the Action Functional}

We now consider small variations $\varepsilon \eta_{\sigma}\left(x_{\mu}\right)$ of the considered fields $\Psi_{\sigma}\left(x_{\mu}\right)$ :

$$
\tilde{\Psi}_{\sigma}\left(x_{\mu}\right)=\Psi_{\sigma}\left(x_{\mu}\right)+\varepsilon \eta_{\sigma}\left(x_{\mu}\right)
$$

where the $\eta_{\sigma}\left(x_{\mu}\right)$ are any set of linearly independent functions of the $x_{\mu}$ which vanish on the part $\partial_{d} T\left(\partial_{d} T \subseteq \partial T\right)$ of the boundary $\partial T$ of the hyper-volume $T$, on which the kinematical condition are prescribed. The variation of the action functional can then be computed as:

$$
\Delta \mathfrak{A}=\int_{T} \mathfrak{L}\left(x_{\mu}, \tilde{\Psi}_{\sigma}, \frac{\partial \tilde{\Psi}_{\sigma}}{\partial x_{\mu}}\right)-\int_{T} \mathfrak{L}\left(x_{\mu}, \Psi_{\sigma}, \frac{\partial \Psi_{\sigma}}{\partial x_{\mu}}\right)
$$

The computation of the variation of the Action Functional now proceeds as follows:

$$
\begin{aligned}
\Delta \mathfrak{A} & =\int_{T} \mathfrak{L}\left(x_{\mu}, \Psi_{\sigma}+\varepsilon \eta_{\sigma}, \frac{\partial \Psi_{\sigma}}{\partial x_{\mu}}+\varepsilon \frac{\partial \eta_{\sigma}}{\partial x_{\mu}}\right) \\
& -\int_{T} \mathfrak{L}\left(x_{\mu}, \Psi_{\sigma}, \frac{\partial \Psi_{\sigma}}{\partial x_{\mu}}\right)+O\left(\varepsilon^{2}\right)
\end{aligned}
$$

Which, with a slight abuse of notations, can be written at the first order in $\varepsilon$ as:

$$
\delta \mathfrak{A}=\varepsilon \int_{T} \sum_{\sigma}\left[\frac{\partial \mathfrak{L}}{\partial \Psi_{\sigma}} \eta_{\sigma}+\sum_{\mu=1}^{m} \frac{\partial \mathfrak{L}}{\partial\left(\partial \Psi_{\sigma} / \partial x_{\mu}\right)} \frac{\partial \eta_{\sigma}}{\partial x_{\mu}}\right]
$$

Integrating by parts and recalling that $\eta_{\sigma}$ vanish on $S$ it is easy to get:

$$
\begin{aligned}
\delta \mathfrak{A} & =\varepsilon \int_{T} \sum_{\sigma} \eta_{\sigma}\left\{\frac{\partial \mathfrak{L}}{\partial \Psi_{\sigma}}-\sum_{\mu=1}^{m} \frac{\partial}{\partial x_{\mu}}\left[\frac{\partial \mathfrak{L}}{\partial\left(\partial \Psi_{\sigma} / \partial x_{\mu}\right)}\right]\right\} \\
& +\varepsilon \int_{\partial T / \partial_{d} T} \sum_{\sigma} \eta_{\sigma} \sum_{\mu=1}^{m} \frac{\partial \mathfrak{L}}{\partial\left(\partial \Psi_{\sigma} / \partial x_{\mu}\right)} N_{\mu}
\end{aligned}
$$

where $\partial T / \partial_{d} T$ is the symmetric difference between $\partial T$ and $\partial_{d} T$ and $N_{\mu}$ is the external unit normal of $\partial T / \partial_{d} T$.

Imposing the stationarity condition

$$
\delta \mathfrak{A}=0
$$

the arbitrariness of $\eta_{\sigma}$ gives, for any $\sigma$ :

$$
\begin{aligned}
\frac{\partial \mathfrak{L}}{\partial \Psi_{\sigma}}-\sum_{\mu=1}^{m} \frac{\partial}{\partial x_{\mu}}\left[\frac{\partial \mathfrak{L}}{\partial\left(\partial \Psi_{\sigma} / \partial x_{\mu}\right)}\right] & =0, \quad \forall x_{\mu} \in T \\
\sum_{\mu=1}^{m} \frac{\partial \mathfrak{L}}{\partial\left(\partial \Psi_{\sigma} / \partial x_{\mu}\right)} N_{\mu} & =0, \quad \forall x_{\mu} \in \partial T / \partial_{d} T
\end{aligned}
$$


In the case of a discontinuity material surface $\Sigma$, with unit normal $N_{\mu}$, the (10) have to completed by

$$
\sum_{\mu=1}^{m}\left[\left|\frac{\partial \mathfrak{L}}{\partial\left(\partial \Psi_{\sigma} / \partial x_{\mu}\right)}\right|\right] N_{\mu}=0, \quad \forall x_{\mu} \in \Sigma
$$

where $[|(\cdot)|]$ is the jump of $(\cdot)$ across the surface $\Sigma$. These equations are known as the Euler-Lagrange equations corresponding to the considered Lagrangian density.

\subsection{The Space-Time Case $\left(\mathbb{R}^{4}\right)$}

Let us now consider the particular case in which $m=4$. This case commonly corresponds to the case $x_{\mu}=\left(x_{1}, x_{2}, x_{3}, t\right)$. We have that $\eta_{\sigma}\left(x_{\mu}\right)$ are any set of linearly independent functions of $x_{\mu}$ which vanish on the boundary of time type domain,

$$
\eta_{\sigma}\left(x_{1}, x_{2}, x_{3}, t_{0}\right)=\eta_{\sigma}\left(x_{1}, x_{2}, x_{3}, t_{1}\right)=0
$$

and on the part $\partial_{d} V$ of the boundary $\partial V$ of the volume $V$, on which the kinematical conditions are fixed,

$$
\eta_{\sigma}\left(x_{1}, x_{2}, x_{3}, t\right)=0, \quad \forall\left(x_{1}, x_{2}, x_{3}\right) \in \partial_{d} V, \quad \forall t \in\left[t_{0}, t_{1}\right] .
$$

It is easy to show that in this particular case equation (7):

$$
\begin{aligned}
\delta \mathfrak{A} & =\varepsilon \int_{t_{0}}^{t_{1}} d t \int_{V} \sum_{\sigma} \eta_{\sigma}\left[\frac{\partial \mathfrak{L}}{\partial \Psi_{\sigma}}-\sum_{k=1}^{3} \frac{\partial}{\partial x_{k}}\left(\frac{\partial \mathfrak{L}}{\partial\left(\partial \Psi_{\sigma} / \partial x_{k}\right)}\right)-\frac{\partial}{\partial t}\left(\frac{\partial \mathfrak{L}}{\partial\left(\partial \Psi_{\sigma} / \partial t\right)}\right)\right] d V \\
& \left.+\varepsilon \int_{\partial V / \partial_{d} V} \sum_{\sigma} \eta_{\sigma} \sum_{k=1}^{3} \frac{\partial \mathfrak{L}}{\partial\left(\partial \Psi_{\sigma} / \partial x_{k}\right)} N_{k}+\int_{\Sigma} \sum_{\sigma}\left[\mid \eta_{\sigma} \sum_{k=1}^{3} \frac{\partial \mathfrak{L}}{\partial\left(\partial \Psi_{\sigma} / \partial x_{k}\right)}\right]\right] N_{k}
\end{aligned}
$$

The stationarity $\delta \mathfrak{A}=0$ of the action implies, for any $\sigma=1,2, \ldots, n$,

$$
\begin{aligned}
\frac{\partial \mathfrak{L}}{\partial \Psi_{\sigma}}-\sum_{k=1}^{3} \frac{\partial}{\partial x_{k}}\left(\frac{\partial \mathfrak{L}}{\partial\left(\partial \Psi_{\sigma} / \partial x_{k}\right)}\right)-\frac{\partial}{\partial t}\left(\frac{\partial \mathfrak{L}}{\partial\left(\partial \Psi_{\sigma} / \partial t\right)}\right) & =0, \forall x_{k} \in V \\
\sum_{k=1}^{3} \frac{\partial \mathfrak{L}}{\partial\left(\partial \Psi_{\sigma} / \partial x_{k}\right)} N_{k} & =0, \forall x_{\mu} \in \partial V / \partial_{d} V(14) \\
\sum_{k=1}^{3}\left[\left|\frac{\partial \mathfrak{L}}{\partial\left(\partial \Psi_{\sigma} / \partial x_{k}\right)}\right|\right] N_{k} & =0, \forall x_{\mu} \in \Sigma
\end{aligned}
$$

Which are the standard Euler-Lagrangian equations. We show below how to generalise (15) when $\Sigma$ can freely move.

\subsection{Rayleigh-Hamilton Principle}

Least Action Principle is not easily adapted to deal with dissipative systems (dell'Isola, Placidi, 2012). A chance to overcome the related difficulties consists in postulating the Rayleigh-Hamilton Principle. It is based on the choice of a dissipation functional which is a functional of the velocities along the motions. 
The first variation with respect to velocities of this functional has to be calculated on the first variations of motions and to be equated to the first variation of Action Functional, to get the searched equations of motions.

One can introduce a dissipation functional of the type

$$
\mathfrak{D}=\int_{T} \mathfrak{R}\left(x_{\mu}, \dot{\Psi}_{\sigma}, \frac{\partial \dot{\Psi}_{\sigma}}{\partial x_{\mu}}\right)
$$

where $\mathfrak{R}$ is the Rayleigh potential density.

The first variation of this functional with respect to $\dot{\Psi}_{\sigma}$ is a linear functional of the variation of its argument. This linear functional has to be calculated in $\eta_{\sigma}$ and equated to $\delta \mathfrak{A}$ in equation 8 to produce the final evolution equations. Remark that the conditions 9 and 10, 11 are correspondingly modified with the introduction of derivatives of $\mathfrak{R}$.

\subsection{Principle of Virtual Work and Principle of Least Ac- tion}

Sometimes it is believed that postulation methods based on the Principle of Virtual Work are much different from those recalled in the previous subsections. This is not indeed the case, as there is a strict relationship between them.

The Principle of Least Action, when formulated for action functionals admitting first differentials, can be regarded as a particular form of the Principle of Virtual Work. If one can decompose the action into its internal, external and inertial parts, as follows

$$
\mathfrak{A}=\mathfrak{A}^{\text {int }}+\mathfrak{A}^{\text {ext }}+\mathfrak{A}^{\text {ine }}
$$

then

$$
\delta \mathfrak{A}=0 \quad \Longleftrightarrow \quad \delta \mathfrak{A}^{\text {int }}+\delta \mathfrak{A}^{\text {ext }}+\delta \mathfrak{A}^{\text {ine }}=0
$$

Identifying the decomposed actions with corresponding powers

$$
\delta \mathfrak{A}^{\text {int }}=\mathfrak{P}^{\text {int }} \quad \delta \mathfrak{A}^{\text {ext }}=\mathfrak{P}^{\text {ext }}, \quad \delta \mathfrak{A}^{\text {ine }}=\mathfrak{P}^{\text {ine }}
$$

we get

$$
\mathfrak{P}^{\text {int }}+\mathfrak{P}^{\text {ext }}+\mathfrak{P}^{\text {ine }}=0
$$

which is the standard form of Principle of Virtual Power, equivalent to the Principle of Virtual Work when action functionals admit first differentials, as mentioned.

\section{State of the Art}

In general, metamaterials are artificial materials engineered by assembling multiple individual elements, usually arranged in (quasi-)periodic patterns, in order to show very peculiar and specific physical properties, due to their designed substructures and not to their composition.

It may be remarked that the expression metamaterial and the early studies in this field were primarily diffused among the scientific area dominated by 
physicists: so first examples of designed metamaterials had very peculiar electromagnetic, optical or thermodynamic properties. Subsequently, the so-called mechanical metamaterials received the attention of scientists: e.g. in Xu et al. (1999), Engheta, Ziolkowski (2006) and Christensen, de Abajo (2012) mechanical exotic properties were conceived and exploited. These materials may also show interesting coupling phenomena with several thermodynamic or electromagnetic unconventional properties (Berezovski, Maugin, 2005a,b; Berezovski, Engelbrecht, Peets, 2010; Berezovski, Engelbrecht, Berezovski, 2011; Berezovski, Engelbrecht, Maugin, 2011; Maugin, Muschik, 1994a,b). These coupling phenomena deserve a great attention as they can be fruitfully developed.

We focus on a small part of the literature on metamaterial: we are interested in so-called micro-structured metamaterials, having a behaviour expressible with continuum models in which along with the placement field other kinematic descriptors are employed. In the rest of this paper we refer to them simply as metamaterial, but otherwise specified. In this context we mention the classical works by Eringen (1999, 2001, 2002), Eringen, Suhubi (1964a,b), Sedov $(1968,1972)$, Toupin $(1962,1964)$ and Bleustein (1967), among those which merit considerable attention.

The idea underlying the theory of micro-structured continua is simple: the complexity of the structure of some materials or living tissues (Federico, Herzog, 2008b,c; Federico, Gasser, 2010; Madeo, Lekszycki, dell'Isola, 2011; Federico, Grillo, 2012; Lekszycki, dell'Isola, 2012) or reinforcements (Nadler, Papadopoulos, Steigmann, 2006; Ferretti et al., 2013) can be still described by considering field theories, but with the addition of tensorial fields to account for the time evolution of micro-structures.

In the formulation of these new models, the problem of determining the most suitable evolution equations arises once the kinematic parameters are selected: in our opinion the most effective method is based on the formulation of a suitable variational principle, as in the references stemming from the fundamental work by Lagrange (Lagrange, 1788), e.g. Auffray et al. (2013), Daher, Maugin (1986), Epstein (2010), Germain (1973a,b), Green, Rivlin (1964a), Kroner (1968), Maugin (2013), Sedov (1968), Toupin (1962,1964) and Bedford (1985).

Indeed, variational methods have the very positive feature to produce wellposed mathematical problems, where the corresponding energy and dissipation functionals are well-behaving, and to lead more easily to the formulation of effective numerical integration schemes.

Of course, in formulation and use of these enhanced field theories, the adoption of Ricci and Levi-Civita indicial notation (Ricci-Curbastro, Levi-Civita, 1900; Lebedev, Cloud, Eremeyev, 2010) and absolute tensor calculus cannot be avoided and it is simply not conceivable to tackle these theories with a component-wise notation. In this context, the differential geometry plays an essential role as it already does in standard continuum mechanics (Epstein, 2010; Epstein, Segev, 1980; Segev, 1986, 2000; Spivak, 1979).

\subsection{Cosserat and micromorphic continua}

About fifty years later Piola's main contributions, his ideas were developed. A Lagrangian density example for Piola-type media is 


$$
\begin{aligned}
& \mathfrak{L}(\nabla \mathbf{u}, \nabla \nabla \mathbf{u}, \dot{\mathbf{u}})=\frac{1}{2} \rho\|\dot{\mathbf{u}}\|^{2}+ \\
& -\left(\mu\|\operatorname{sym} \nabla \mathbf{u}\|^{2}+\frac{\lambda}{2}(\operatorname{tr} \nabla \mathbf{u})^{2}+\frac{\alpha}{2}\|\nabla \nabla \mathbf{u}\|^{2}\right)
\end{aligned}
$$

where $\rho$ is the mass density, $\mu$ and $\lambda$ are the Lamé parameters, $\alpha$ is the second gradient stiffness and $\mathbf{u}$ is the displacement field.

The Cosserat brothers were among the first authors who complemented, with additional independent kinematic fields, the standard kinematics constituted by a placement field. According to them, these fields are Euler angles, representing rigid rotations of the microstructure with respect to the overall continuum local displacement. Cosserat contributions (Cosserat brothers, 1909) were underestimated for other fifty years and only starting from 1960 a group constituted by notable scientific personalities as Mindlin, Green and Rivlin, Toupin, Eringen and Germain (Green, Rivlin, 1964a,b,c, 1965; Mindlin, 1964, 1965; Toupin, 1962, 1964; Eringen, 1999, 2001, 2002; Eringen, Suhubi, 1964a,b; Germain, 1973a,b) managed to establish, yet with some resistances, the validity of Cosserat's point of view.

Actually, Cosserat's approach can be further generalised including in macroscopic models, along with micro-rotations, also micro-stretches, micro-strains or concentrated micro-deformations, so introducing the so-called micro-structured or micropolar or micromorphic continuum models (Erofeyev, Potapov, 1993; Potapov, Pavlov, Maugin, 1999; Erofeyev, Pavlov, Leontiev, 2013). These can be formulated through a postulation process based on the principle of least action (Auffray et al., 2013) or on the principle of virtual works (Maugin, Metrikine, 2010; Maugin, 2013). Later on, alternative postulation procedures, based on generalised balance laws, have been also attempted (Eringen, 1999, 2001).

Indeed, as already remarked in Piola (1846), it is rather unlikely to achieve a standard Cauchy continuum starting from a discrete system with a multilength-scale microstructure.

The evolution of non purely mechanical phenomena can be described within the framework of micromorphic continuum theory: in this context we refer e.g. to phenomena observed in piezoelectromechanical structures (Vidoli, dell'Isola, 2000, 2001) where also electromagnetic descriptors are necessary to characterise the kinematics of the system.

The micromorphic model, as a natural generalisation of the Cosserat approach, has also mainly been studied in its linearised version. Existence results for the nonlinear static setting are quite recent (Neff, 2006c; Neff, Forest, 2007). There are manifold connections to models of gradient continua and elasto-plasticity, still awaiting closer mathematical inspection (Neff et al., 2009; Klawonn, 2011).

It has to be explicitly remarked that Piola's and Cosserat's models can be reconciled, i.e. Cosserat reduced to Piola, introducing internal constraints and Lagrange multipliers as clearly stated in (Bleustein, 1967): the second gradient materials can be seen as a particular limit case of the micromorphic media as they can be obtained from micromorphic ones constraining the micromorphic tensor to be equal to the classical strain tensor. Actually one can get Piola's deformation energies depending on higher gradients of displacement, as a limit of many different, physically non-equivalent, more detailed micromorphic models. 
To formulate three-dimensional Cosserat theories, the framework built to construct Cauchy's model needs to be deeply modified and in particular the concept of stress needs to be revisited (Neff, 2006a,b; Neff, Jeong, 2009; Pietraszkiewicz, Eremeyev, 2009). This last difficulty is a serious conceptual one, as Cauchy paradigm has become a mental habitude, or even a natural belief, for many engineers and material scientists: as a matter of fact, in the opinion of the authors, the first scientists who managed to get rid of it were actually physicists.

A Lagrangian density example for Cosserat-type media can be expressed

$$
\begin{aligned}
& \mathfrak{L}(\nabla \mathbf{u}, \mathbf{Q}, \dot{\mathbf{u}}, \dot{\mathbf{Q}})=\frac{1}{2} \rho\|\dot{\mathbf{u}}\|^{2}+\frac{1}{2} \eta\|\dot{\mathbf{Q}}\|^{2}+ \\
& -\left(\mu_{e}\|\operatorname{sym} \nabla \mathbf{u}\|^{2}+\frac{\lambda_{e}}{2}(\operatorname{tr} \nabla \mathbf{u})^{2}+\mu_{c}\|\operatorname{skew}(\nabla \mathbf{u})-\mathbf{Q}\|^{2}+\frac{\alpha}{2}\|\operatorname{Curl}(\mathbf{Q})\|^{2}\right)
\end{aligned}
$$

where $\nabla \mathbf{u}$ is the gradient of displacement field and the micro-rotation tensor $\mathbf{Q}$ is a second order tensor associated to the microstructure rotations, $\rho$ is the density per unit of macro volume of the material, $\eta$ is the micro-density and $\mu_{e}, \lambda_{e}, \mu_{c}$ and $\alpha$ are elastic coefficients assumed to be constant.

The Cosserat brothers never proposed a linearisation of their intrinsically nonlinear theory, neither elaborated on any specific constitutive law. The rediscovery of the Cosserat approach in the second half of the last century was mainly restricted to a linearisation of their equations. However, more than 50 years later, no material has been conclusively shown to be a Cosserat material yet. For the linear Cosserat model and its mathematical complexity we refer to the discussion in Neff, Jeong (2009) and Neff, Jeong, Fischle (2010). Devoid of any physical meaning, the linear Cosserat approach may be used as a regularisation for otherwise ill-posed problems, e.g. in elasto-plasticity (Neff, Wieners, 2003; Neff, Chełmiński, 2005).

The problems with the linear Cosserat model, referred to above, are all connected to the question of how the Cosserat rotations and the macroscopic rotations are locally coupled. This coupling is governed in the linearised model by the Cosserat couple modulus $\mu_{c}$, which penalises the quadratic difference between micro- and macro-rotation. Its numerical value is under constant debate. We run into the role of that modulus later on, again in conjunction with the micromorphic models.

A new turn for the Cosserat model can be found in the Neff's work. With a closer look to the original nonlinear Cosserat model, he provided the first existence proof in the geometrically nonlinear case and investigated some physical applications. As it turned out, the question of how to couple micro- and macro-rotations in the nonlinear model has different answers. One may use the already known linear coupling, relying on the introduction and determination of the Cosserat couple modulus $\mu_{c}$, or one may resort to a truly nonlinear coupling, as strongly favoured in Neff (2004b) and Münch, Wagner, Neff (2011), in which a linearisation would turn into classical elasticity without Cosserat effects. The nonlinear coupling involves much more complicated mathematics which require deeper insights in the analytical aspects (Neff, 2002; Neff, Münch, 2008; Neff, Pompe, 2013).

Let us finally mention a Cosserat success story: the application of the concept in the realm of plates and shells. Here, the introduction of an or- 
thogonal frame to the shells surface is most natural and is the preferred choice from an engineering point of view. The first existence results for the geometrically nonlinear Cosserat plate model has been proposed again by Neff and subsequently extended to shells (Neff, 2004a; Bîrsan, Neff, 2013).

\subsection{Acoustic metamaterials and wave propagation in mi- cromorphic continua}

The concept of acoustic metamaterials is attracting increasingly the interest of physicists and mechanicians. It is described and studied in many works: we refer here e.g. to Engheta, Ziolkowski (2006) or Zouhdi, Ari, Vinogradov (2008).

In this field, the particular shape, geometry, size, orientation and arrangement of metamaterial constitutive elements can affect the propagation of light or sound waves in a not-yet-observed manner, creating material properties which cannot be found in nature.

Particularly promising in the design of metamaterials are those with microstructures which present high-contrast in microscopic properties: these highly heterogeneous microstructures, once homogenised, produce generalised continuum models (Alibert, Seppecher, dell'Isola, 2003; Forest, Sievert, 2006; Forest, 2009; Misra, Ching, 2013; Misra, Singh, 2013). In these metamaterials, when the size of the Representative Elementary Volume (REV) tends to zero, some of the physical micro-properties characterising the behaviour diverge, while simultaneously some others vanish, although the micro-structures remain quasiperiodical.

One has to remark that the standard homogenisation techniques need to be modified and/or generalised in order to be adapted to the process of identifying the macro-properties of metamaterials (Steinmann, Elizondo, Sunyk, 2007; Sunyk, Steinmann, 2003; Nadler, Papadopoulos, Steigmann, 2006; Misra, Singh, 2013; McBride et al., 2012; dell'Isola, Rosa, Woźniak, 1997, 1998; Andreaus, Ruta, 1998).

In literature, attention has been drawn on a particular mechanical metamaterial sub-class, which is significant to the considerations of this paper: the acoustic metamaterials. To give a hint of the possible applications of the newly designed metamaterials, we list some papers which are more relevant to our results, especially in the perspective of their extension to $2 \mathrm{D}$ and $3 \mathrm{D}$ systems. In Lee et al. (2010) a composite medium, exhibiting negative effective bulk modulus or negative effective mass density or both of them, is studied. In Kolpakovs (1985) and Xu et al. (1999) materials with negative Poisson's ratio, named auxetic materials, were designed. Anisotropic versions of the so-called pentamode structures, i.e. having a finite bulk modulus and vanishing shear modulus, are candidates for effective control of acoustic waves (Christensen, de Abajo, 2012). In Porubov (2003) amplification of strain waves are investigated.

The papers Neff et al. (2013) and Ghiba et al. (2013) study a large class of evolution equations governing the propagation of linear waves in micromorphic or generalised continua (Placidi et al., 2013; Rosi, Giorgio, Eremeyev, 2013). A series of papers (Neff, Pauly, Witsch, 2012, 2013), which propose the mathematical bases necessary for the formulation of the new relaxed micromorphic model, are of independent interest.

The Lagrangian density for the relaxed micromorphic continuum in isotropic, linear-elastic case, as proposed in Ghiba et al. (2013) and Neff et al. (2013), can 
be introduced

$$
\begin{aligned}
& \mathfrak{L}(\mathbf{u}, \nabla \mathbf{u}, \mathbf{P}, \dot{\mathbf{u}}, \dot{\mathbf{P}})=\frac{1}{2} \rho\|\dot{\mathbf{u}}\|^{2}+\frac{1}{2} \eta\|\dot{\mathbf{P}}\|^{2}+ \\
& -\left(\mu_{e}\|\operatorname{sym}(\nabla \mathbf{u}-\mathbf{P})\|^{2}+\frac{\lambda_{e}}{2}(\operatorname{tr}(\nabla \mathbf{u}-\mathbf{P}))^{2}+\mu_{h}\|\operatorname{sym} \mathbf{P}\|^{2}+\frac{\lambda_{h}}{2}(\operatorname{tr} \mathbf{P})^{2}+\right. \\
& \left.+\mu_{c} \| \text { skew }(\nabla \mathbf{u}-\mathbf{P})\left\|^{2}+\frac{\alpha_{c}}{2}\right\| \operatorname{Curl} \mathbf{P} \|^{2}\right)
\end{aligned}
$$

where $\mathbf{u}$ is the displacement field and the microstrain tensor $\mathbf{P}$ is a second order tensor which accounts for deformations associated to the medium microstructure, $\rho$ is the density per unit of macro volume of the material, $\eta$ is the micro-density and $\mu_{e}, \mu_{h}, \mu_{c}, \lambda_{e}, \lambda_{h}$ and $\alpha_{c}$ are elastic coefficients assumed to be constant.

It is clear that, in further investigations, the great variety of propagating waves that may exist in micromorphic or complex continua may unfold useful engineering applications, through the design of particularly tailored metamaterials with up-to-now not imagined features. The problems studied in Neff (2002) and Neff, Jeong (2009) are the mathematical basis for these developments.

We already mentioned that Bleustein (Bleustein, 1967) shows how Cosserat's model reduces to Piola's. In this sense, the results obtained from the study of wave propagation in micromorphic media intrinsically contains those of second gradient media. Introductory results on wave propagation in second gradient elastic media have shown a variety of exotic phenomena in mainly shielding or transmitting through embedded media interfaces. It has been shown that (dell'Isola, Madeo, Placidi, 2012; Placidi et al., 2013; Rosi, Madeo, Guyader, 2013; Rosi, Giorgio, Eremeyev, 2013), for waves at frequencies sufficiently high to interact with the microstructure, the shielding or transmitting properties can be significantly enhanced. Technologically significant devices may be designed exploiting such exotic wave propagation properties, for example in the fields of vibration and acoustic passive control or stealth technology. Some preliminary results on the study of wave propagation in micromorphic media indicate that, for particular sets of the constitutive parameter values, propagation of some types of waves can be inhibited or waves that propagate without carrying energy can be also observed. Such exotic frequency-dependent behaviours are observable as bulk properties of the micromorphic medium, ignoring more complicated reflection and transmission phenomena at the surfaces of material properties discontinuity. This means that well-conceived micromorphic materials may be used as exotic wave-guides which allow, depending on the envisaged use, to filter and switch on and off some typical waves.

\subsection{Functional Structural modifications and their use for designing metamaterials}

In the mechanics of materials and structures the concept of "functional structural modification" has been developed as a modification of a principal structure, without changing its original uses, aimed at obtaining an engineering collateral purpose, generally an improvement of the behaviour (Carcaterra, 2005; Carcaterra, Akay, Ko, 2006; Carcaterra, Akay, 2007, 2011; Altenbach, Eremeyev, 
2008, 2009; Bîrsan et al., 2012).

Relevant examples of principal structures are systems for carrying loads in space applications and industrial or space robotic arms affected by forced vibrations that, in absence of damping, could eventually have a destructive effect. In these cases, structural modifications of the principal structures are aimed at the addition of some elements that damp vibrations: these devices may be e.g. arrays of piezoelectric actuators coupled with dissipative electronic circuits (dell'Isola, Vidoli, 1998b).

In general, a structural modification is the addition of a designed new structure which -once coupled with the original one- will produce the desired, efficient effect.

It is our opinion that multidisciplinary studies are very often fruitful and crucial in technology advancement. On the contrary, a lost awareness in engineering sciences concerns the importance of scientific knowledge in the process of conceiving technological applications; interesting considerations on this subject are in (Russo, 2004).

In this context it has to be remarked that the very interesting concepts that motivated the papers Carcaterra, Sestieri 1995, Carcaterra et al. 2000, Culla, Sestieri, Carcaterra 2003, Carcaterra 2005 and Carcaterra, Akay 2007 stem from the thermodynamics and statistical mechanics of irreversible systems. Although the theory of mechanical vibrations is often considered far from thermodynam$\mathrm{ics}^{1}$, these papers show how the concepts, that one may consider very distant, of irreversibility, Poincaré recurrence time and thermodynamic equilibrium are prolific in this context. Actually in those papers, the concept of structural modification is considered together with the concept of hamiltonian system and internal degrees of freedom: a structure is coupled with many internal extra degrees of freedom properly designed. In this way, one can trap mechanical vibration energy for a very long time, related to the Poincaré recurrence time, and thus construct a structure with an effective damping system.

Alternatively, it will be an interesting challenge to design metamaterials whose material particles are endowed with such energy sinks. These sinks may be conceived as some growing micro-structural damage (dell'Isola, Woźniak, 1997a; Misra, Singh, 2013; Rinaldi, Lai, 2007b; Rinaldi, 2013; Yang, Misra, 2010, 2012; Yang, Ching, Misra, 2011; Auffray, Bouchet, Brechet, 2010). In this case, until the evolution of micro-damages do not affect the macroscopic material behaviour, the applied loads is sustained, as the design requires. Of course a micro-structural yield criterion has to defined to assure the macrostructural integrity of the metamaterial. This topic is further discussed in some of the following sections.

\section{Smart Structures with distributed control}

A class of metamaterials is represented by controlled smart structures, recently introduced to optimise the dissipation of mechanical vibration energy. The purpose is to tune an electronic circuit network in such a way that the electric eigenfrequencies always coincide with the mechanical eigenfrequencies of the structural member to be damped.

\footnotetext{
${ }^{1}$ Very often mechanical engineers specialise their skills, alternatively, focusing on or simply ignoring thermodynamics.
} 
This process is based on the known equivalence concept between structural members and analogue circuits. The investigations in this field were developed in the period 1940-1970 for another engineering application, i.e. the design of analogue computers: the knowledge acquired in that field can be exploited with these other purposes.

In dell'Isola, Vidoli (1998a,b), Vidoli, dell'Isola (2000, 2001), Andreaus, dell'Isola, Porfiri (2004), Maurini, dell'Isola, Del Vescovo (2004), Maurini, Pouget, dell'Isola (2004, 2006), Porfiri, dell'Isola, Santini (2005) and Giorgio, Culla, Del Vescovo (2009) the piezoelectric transduction is applied to damp vibrating shells or beams by means of a multimodal, passive control, obtained designing and coupling a distributed electronic circuit with exactly the same evolution equations as those pertaining the structural member.

These metamaterials are based on the following concept: the dynamic evolution of the structural members is linked via piezoelectric coupling terms, whose mathematical structure is that of gyroscopic coupling, to the dynamic evolution of the micro-structure parameter. The latter is physically associated to observable electric potentials of a designed electronic circuital network, whose characteristics can be theoretically determined and physically realised. Evidently, the quality of precision, design and construction of electronic devices cannot be paralleled by mechanical ones.

It is reasonable to assume that the Lagrangian density of the homogenised piezo-composite plate is (Alessandroni et al., 2005)

$$
\begin{aligned}
& \mathfrak{L}(u, \dot{u}, \dot{\psi})=\frac{1}{2} \rho_{t} \dot{u}^{2}+\frac{1}{2} c \dot{\psi}^{2}-g \dot{\psi}\left(\frac{\partial^{2} u}{\partial x_{1}^{2}}+\frac{\partial^{2} u}{\partial x_{2}^{2}}\right)+ \\
& -\frac{1}{2} S_{t}\left[\left(\frac{\partial^{2} u}{\partial x_{1}^{2}}\right)^{2}+\left(\frac{\partial^{2} u}{\partial x_{2}^{2}}\right)^{2}+2 \nu_{t} \frac{\partial^{2} u}{\partial x_{1}^{2}} \frac{\partial^{2} u}{\partial x_{2}^{2}}+2\left(1-\nu_{t}\right) \frac{\partial^{2} u}{\partial x_{1} \partial x_{2}}\right]
\end{aligned}
$$

in which $\rho_{t}$ is the effective mass per unit surface, $S_{t}$ is the effective bending stiffness, $\nu_{t}$ is the effective Poisson ratio, $c$ is the effective capacitance per unit surface, $g$ is the effective piezoelectric coupling and $u$ is the transversal displacement.

The metamaterial is globally behaving as an electromechanical wave-guide where the electric part is an internal resonator coupled to an oscillator of the mechanical subsystem.

From a purely mathematical point of view, one observes the following circumstances: i) the vibration spectrum of both the mechanical structure and its electric counterpart are exactly the same and ii) the electric system is thought as a mirror system whose evolving parameter is the micro-structural kinematic descriptor. Hence, piezoelectromechanical structures are particular micro-structured continua of the kind studied e.g. by Toupin, Eringen and Mindlin (Toupin, 1962, 1964; Eringen, 1999, 2001; Mindlin, 1964; Mindlin, Eshel, 1968).

Since piezoelectromechanical structures are controlled systems, the microstructure is designed as an optimal controller which maximises the mechanical energy absorption and possibly dissipate it through dampers i.e. electric resistances.

It has to be remarked that, in the mentioned investigations, shells or more complex structures are not treated neither nonlinear cases are studied, i.e. only 
material and geometric linear, simple systems are considered. So, it could be very interesting to investigate the behaviour of piezoelectromechanical structures in the case of nonlinear and more complex structural members.

Moreover, these metamaterials present some stability and bifurcation problems, especially when follower forces are considered. In a few papers (Pignataro, Luongo, 1987; Michel, Limam, Jullien, 2000; Jamal et al., 2003; Luongo, Di Egidio, 2005; Luongo, Romeo, 2006; Belyakov, Seyranian, Luongo, 2009; Luongo, Zulli, Piccardo, 2009; Limam et al., 2010), for cases of similar complexity, these difficulties are dealt with. A preliminary study, reported in a private communication by A. Luongo, seems to indicate that piezoelectromechanical structures are less stable, in a way not yet rigorously specified, than their original mechanical counterpart. Hence, it should be planned a series of theoretical, numerical and experimental analyses of the bifurcation of piezoelectromechanical structures, using the methods presented e.g. in Luongo (2001), Paolone, Vasta, Luongo (2006) and Di Egidio, Luongo, Paolone (2007), aimed at characterising or even exploiting their possible imperfections and at identifying some of their constitutive parameters.

\subsection{Microstructured continua as damaged body models}

Bodies with micro-cracks or other damage mechanisms can be modelled by means of either discrete methods, producing finite dimensional models, or homogenised models with extra kinematical descriptors.

Interesting results in this context can be found in Rinaldi, Lai (2007a,b), Rinaldi, Krajcinovic, Mastilovic (2007),Rinaldi et al. (2008) Rinaldi (2009), Yang, Misra (2010) and Misra, Singh (2013), where the final goal is to produce a macroscopic field theory based on the knowledge, not necessarily detailed, of the micro-structure of the mechanical system.

To model the damage mechanisms, Eringen continua (Eringen, 1999, 2001, 2002; Eringen, Suhubi, 1964a,b; Sedov, 1968, 1972; Kroner, 1968) have to be assumed with the addition of dissipative effects, e.g. via Hamilton-Rayleigh dissipation potentials, to take into account the irreversible phenomena involving dissipation of energy.

The phenomena of material transformation due to the damage evolution are similar to phase transitions (dell'Isola, Romano, 1987b; dell'Isola, Woźniak, 1997a,b; Eremeyev, Freidin, Sharipova, 2003), with the addition of irreversibility (Cuomo, Contrafatto, 2000; Contrafatto, Cuomo, 2005). Damage may be described by plastic deformation phenomena (Contrafatto, Cuomo, 2002, 2006; Forest, 2009; Ciancio, Carol, Cuomo, 2006, 2007) or by crack growth (Andreaus, Baragatti, 2009).

A vibration control may be obtained by degrading part of the mechanical energy involved in some micro-structural vibrations by means of the dissipation associated to damage phenomena, like e.g. plastic deformation. This control can be fruitfully employed as long as the provoked structural modifications do not compromise the mechanical properties of the structure.

\subsection{Metamaterials designed by species evolution}

It has already happened in science that a new field of investigation has taken advantage of the parallel between structures existing in Nature and human 
designed devices.

A discussion of this deep and interesting topic is not within the scope of this review: we simply cite here the case of Cybernetics, as an exemplary one.

Exotic behaviour actually means only not-yet-studied behaviour. At present, materials that are studied and used in the engineering and technology of the XX century are simple, with the meaning of being sufficiently well modelled with the paradigm of Cauchy continuum mechanics.

However, those scientists who tackled the problem of studying living materials understood that specific tools, much more sophisticated than those used up to that moment, were necessary. With this respect, it is illuminating the pioneering work by Wolff (Wolff, 1892).

The problem of modelling the mechanical behaviour of growing tissues has been confronted by many researchers: in this context we refer to those belonging to two groups.

The first one addresses the problem of the bone growth and reconstruction with the insertion of a graft of artificial bio-resorbable material (Mullender, Huiskes, 1995; Madeo, Lekszycki, dell'Isola, 2011; Lekszycki, dell'Isola, 2012; Madeo et al., 2012). The second group regards the mechanical description of biological tissues, with a special emphasis on their micro-structural behaviour (Federico, Grillo, Herzog, 2004; Federico et al., 2005; Federico, Herzog, 2008a,b,c; Federico, Gasser, 2010; Rinaldi, 2011; Federico, Grillo, 2012; Bellini, Di Martino, Federico, 2013).

The first group of papers is based on the biological knowledge according to which both the growth and resorption of bone tissue is driven by a stimulus depending on external mechanical loads. Various models are introduced in the literature to describe how the stimulus is related to the mechanical excitation and governs bone remodelling, in a living or reconstructed tissue. While in some papers (Mullender, Huiskes, 1995) the stimulus is assumed to be affected by the local state of mechanical deformation and maybe by the its time history, there is biological evidence that also the neighbouring deformation is involved. This circumstance plays a dominant role in the phenomena of growth occurring in reconstructed bones, so needs to be considered. This is done (Madeo, Lekszycki, dell'Isola, 2011; Lekszycki, dell'Isola, 2012; Madeo et al., 2012) introducing integral operators in order to represent the stimulus $S$, at point $\mathbf{x}$ and time $t$, as a linear functional depending on the sensor cell density $d$ and the strain energy $U$ :

$$
S(\mathbf{x}, t)=\int_{-\infty}^{t} \int_{V_{t}} K(\mathbf{x}, \mathbf{y}, t, \tau) U(\mathbf{y}, \tau) d(\mathbf{y}, \tau) d \mathbf{y} d \tau-S_{0}(\mathbf{x}, t)
$$

where the kernel $K(\mathbf{x}, \mathbf{y}, t, \tau)$ represents the range of influence of sensor cells in space and time and $V_{t}(\mathbf{x}, t)$ is the volume of the bone tissue in the current configuration; $S_{0}$, possibly depending on the position and time, is the threshold value of stimulus associated with a biological equilibrium state for which the effect of bone resorption and synthesis are balanced. In the other words, $S$ represents the non-local behaviour both in time and space, like in the viscoelasticity or non-local elasticity (Eringen, Edelen, 1972; Haseganu, Steigmann, 1996; Parks et al., 2008; Lehoucq, Silling, 2008). If the signal from sensor cells is assumed instantaneously transmitted, i.e. the transmission time scale is negligible if compared with the characteristic time of the remodelling phenomena, a simpler 
assumption can be stated

$$
S(\mathbf{x}, t)=\int_{V_{t}} k(\mathbf{x}, \mathbf{y}) U(\mathbf{y}, t) d(\mathbf{y}, t) d \mathbf{y}-S_{0}(\mathbf{x}, t)
$$

where the function $k(\mathbf{x}, \mathbf{y})$ is the reduced form of the range of influence of sensor cells.

The second group of aforesaid papers regards the models for complex biological tissues, considered as composite materials. For instance, some tissues have an internal structure in which a porous matrix filled with an interstitial fluid is reinforced by impermeable collagen fibres: the modelling of these systems is motivated by the need to describe the phenomena related to interstitial fluid flow in articular cartilage. For this purpose, the results in Misra, Chang (1993), Quiligotti, Maugin, dell'Isola (2003),Sciarra, dell'Isola, Coussy (2007), dell'Isola, Madeo, Seppecher (2009),Madeo, Gavrilyuk (2010), Misra, Yang (2010) and Madeo et al. (2013) are useful to model the flow of a compressible or incompressible fluid in deformable porous media. Some difficulties arise in this innovative application field of micro-structured continuum mechanics: we mention for instance, in fibre-reinforced composite materials, the problem to model the permeability both in the initial undeformed configuration and its modifications during the deformation evolution. In this context, the analysis of involved length scales is crucial, as length scale separation allows simplification in the modelling procedure. In any case, different complex homogenisation methods need to be used and very often the capillary properties of the interstitial fluid may be significant: in this case the results developed in Gatignol, Seppecher (1986), Seppecher (1987, 1988, 1989, 1993, 2001), dell'Isola, Seppecher (1995), dell'Isola, Gouin, Seppecher (1995) and dell'Isola, Seppecher, Madeo (2012) can be of use.

\section{Some research perspectives}

The hints sketched in this section are limited to some research developments in progress at the M\&MoCS International Research Centre. The experimental part of investigations, proposed or in progress, need great efforts, since the sophisticated models involved are able to predict unconventional phenomena and thus most laboratory apparatuses are not designed for them.

Great care has to be paid to the theoretical aspects of proposed investigations and their design.

\subsection{Sound and vibration control via the conception of fre- quency filters or enhanced damping effects}

Acoustic metamaterials can be devised employing various phenomena. For a comprehensive description of the necessary models in this context we refer to Maugin, Metrikine (2010).

It is opinion of the authors that the most fruitful models and effects to be exploited are those involving the energy trapping of macroscopic waves in microscopic degrees of freedom, i.e. those which describe more accurately micromotion. The reader should note that in the present context models are preceding 
phenomena, as we look for materials having some peculiar phenomena: this is the commonly accepted point of view when dealing with metamaterials.

The results described in Narisetti, Ruzzene, Leamy (2011, 2012) and Manktelow, Leamy, Ruzzene (2013a) are a useful starting point to enclose non-linearities. Multiple time-scale analyses (Luongo, Paolone, Di Egidio, 2003; Luongo, Di Egidio, 2005) can be used to design wave modulations with energy transfer among vibration modes, the so-called energy spillover, aimed at an effective dissipation. In this case, the active degrees of freedom are those described by the micro-structural kinematical descriptors.

It is clear that, due to the coupling phenomena involving the micro-structure and thank to non-linearities, it is possible to design metamaterials in which there are gaps in wave propagation for selected frequency bands.

On the other hand, in Ghiba et al. (2013) and Neff et al. (2013) it is described a behaviour which is somewhat surprising: for a singular kind of linear micro-structured continuum, not admissible according to Mindlin, it is possible to choose the constitutive parameter values in order to obtain even wide frequency wave propagation gaps. The novelty of this result is twofold: i) it seems that non-linearities are not required to achieve such gaps ii) the mathematical well-posedness, presented by the authors, indicates that the implementation of these metamaterials is highly feasible. Micro-structured continua and their physical feasibility deserve specific investigations, also by means of suitable electronic waveguides. The aforementioned preliminary results promise a rich variety of behaviour for Mindlin-type materials which could provide amazing developments.

Other investigations concerns the invention of metamaterials undergoing phase transitions when solicited by a proper mechanical action: they are named thixotropic materials (Barnes, 1997). Metamaterial undergoing solid-solid phase transitions could be used to reduce obnoxious mechanical vibrations, in the design of acoustic noise shields: the mechanical energy associated with the vibrations causes an endo-energetic phase transition, producing a dissipative effect (dell'Isola, Woźniak, 1997b; Yeremeyev, Freidin, Sharipova, 2007).

Of course, in the description of these phenomena, strong non-linearities and non-convexities commonly occur and mathematical formulation could require higher gradient or micro-structured continua.

Efficient and volume dissipation effects, occurring in porous deformable solids, are an important class of phenomena (Sciarra, dell'Isola, Hutter, 2001; Quiligotti, Maugin, dell'Isola, 2002, 2003; dell'Isola, Guarascio, Hutter, 2000; Altenbach, Eremeyev, 2009). An interesting case takes place when the porous material is partially saturated with compressible fluid whose deformationinduced viscous flow causes energy dissipation (Madeo, dell'Isola, Darve, 2013). The fluid can be totally or partially trapped in the pores, with effects linked to the tortuosity (dell'Isola, Rosa, Woźniak, 1997, 1998; dell'Isola, Hutter, 1998; Sciarra, dell'Isola, Hutter, 2001; Madeo, Gavrilyuk, 2010; Madeo et al., 2013; Rosi, Madeo, Guyader, 2013). The design of metamaterials with fluid inclusions, completely or partially interconnected, or totally or partially isolated, with possibly channel opening mechanisms which dissipate energy, is really promising: slow and fast Biot-type waves may enhance the effects of wave propagation lack in multiple frequency ranges, as already described. 


\subsection{Conception of noise shields}

The design of noise shields through metamaterial characteristics is related to the subject of section 5.1.

Shields are usually modelled by means of bi-dimensional continua. The theoretical analysis of bi-dimensional continua endowed with micro-structure has recently received a great impulse: we refer to the results in Neff (2004a, 2007) and Neff, Chełmiński (2007) for Cosserat shells and in Eremeyev, Zubov (2007), Eremeyev, Altenbach, Morozov (2009) and Eremeyev, Pietraszkiewicz (2011) for micro-structured shells in which phase transition may occur.

These shells are designed with properties capable to stop noise: generalising some results already presented (Madeo, Gavrilyuk, 2010; Madeo et al., 2013; dell'Isola, Madeo, Placidi, 2012; Rosi, Madeo, Guyader, 2013; Rosi, Giorgio, Eremeyev, 2013; Placidi et al., 2013) one can expect to get important technological applications. Again, the key idea is trapping vibration energy in the internal degrees of freedom, i.e. those of the micro-structured shells.

Another class metamaterials may spring up from the comparative analysis between the pre-stressed microstructured shells in Altenbach, Eremeyev (2009, 2010), Altenbach, Eremeyev, Morozov (2009, 2010, 2012) and Altenbach, Eremeyev, Lebedev (2011), and those involving shells with phase transitions in Eremeyev, Pietraszkiewicz (2004, 2006), Eremeyev, Zubov (2007), Pietraszkiewicz, Eremeyev, Konopinska (2007) and Eremeyev, Lebedev (2013). The analysis performed in Placidi et al. (2013) implies that the interfacial mechanical properties may have very interesting and effective benefits on wave transmission and reflection: therefore one can expect that pre-stressed shells, maybe undergoing phase transitions, are efficient noise shields in a wide range of frequencies and amplitudes.

Dissipative contact phenomena involving impact (Cuomo, Ventura, 1998; Andreaus, Placidi, Rega, 2010) or phenomena related to so-called energetic boundaries (Steigmann, Ogden, 1997; Steeb, Diebels, 2004; Steinmann, 2008; Altenbach, Eremeyev, Lebedev, 2010; Altenbach, Eremeyev, 2011; McBride et al., 2011) could also be fruitful. Near future researches may involve the formulation of a three-dimensional boundary value problem with boundaries characterised by one or more of those surface micro-structures. The structure of these boundary conditions may induce

- a time delay in the release of incident energy at the interface,

- a concentrated dissipation at the interface,

- a considerable increase of reflected energy, also with time delay,

- a surface trapping of incident energy with its release in different forms, which could have non-parasitic uses,

- the origination of surface waves, involving surface displacement, surface thickness variation or, more generally, surface micro-structural oscillations.

In the opinion of the authors the devices conceived up to now in general did not try to completely exploit the synergistic effect of all previously listed boundary effects: it seems unavoidable to use all of them simultaneously if one wants to stop a large amount of energy in a very narrow space region. 
Shells are surfaces always constituted by the same set of material particles during the involved phenomena. The modelling difficulties dramatically increase (dell'Isola, Romano, 1986, 1987a,b) when i) the interfaces must be endowed with some material properties, i.e. simultaneously mass, energy, linear momentum, electric charge and so on, and ii) they are constituted by different material particles in different time instants. Actually, materials with phase transition, in presence of mechanical vibrations, exhibit the onset of internal boundary layers (Yeremeyev, Freidin, Sharipova, 2007; Pietraszkiewicz, Eremeyev, Konopinska, 2007; Ferretti et al., 2013), with localisation not known a priori: the free-moving boundary problems that arise are also very difficult to solve, but may produce another effective method for controlling the energy transfer in metamaterials.

\subsection{Metamaterials self-limiting and self-detecting effects of damage}

Damages in materials are often considered as a negative byproduct of motion or external actions. In our opinion this is not always the case, as damaging phenomena, when suitably controlled, may have a positive role in conception of novel engineering devices: one can accept to sacrifice a part of mechanical resistance in order to limit the dynamical effects on a structure. This sacrifice is even more effective if the structure is endowed with a self-healing or selflimiting control. One of the most challenging fields which open to pioneering investigations is exactly the search of such kind of controls.

It has been discussed how this effect may conversely result in some positive microscopically driven damping effects, at least until the damage progress does not cause a permanent structural failure. Healing is that process, occurring in living tissues, of repairing the effects of the progress of damage. A great scientific and technological challenge is the conception of self-healing materials. More modestly, one could imagine systems that are able to self-limit the damage effects and so to continue to be operative also in presence of serious damages.

Piezoelectromechanical systems, described in section 4, belong to this category. These structures, in an initial stage of crack formation in their mechanical part, may continue to correctly operate if the piezoelectric transduction transform enough mechanical energy into its electrical counterpart. The range of validity of this statement needs to be investigated. In particular it has to be assessed the degree of damage

- in the mechanical structure which causes the whole system failure,

- in the transduction system which affects an effective transduction,

- in the electrical part of the wave-guide which makes the piezoelectric transduction useless.

Moreover, the systems with electric and mechanical coupling have a peculiarity: it is possible to use the efficiency and sensitivity of electronic circuits to assess, with periodic electrically driven checks or even observing possible malfunctioning of the electric wave-guide, the status and progression of damage. One can easily design a system of collection and processing of signals in the electric network and interpret possible anomalies, compared with analogous results of numerical simulations, for the damage assessment. 


\subsection{Detection of material properties in growing or recon- structed bone tissue with acoustic techniques}

There are so many similar applications of sonic or ultrasonic techniques that it is not meaningful nor useful to give references about the subject. Here, as an example, a single topic by one of the authors (Del Vescovo, Fregolent, 2005, 2009 ) is cited, in which the problem of detecting damage in frescoes with nondestructive procedures is addressed.

In many papers (Madeo, Lekszycki, dell'Isola, 2011; Lekszycki, dell'Isola, 2012; Andreaus, Giorgio, Lekszycki, 2013) the growth in bone tissue, possibly consequent to reconstruction with bio-resorbable materials, is studied with particular attention to determine the final bone properties. Luckily, ultrasound propagation are greatly influenced by bone mechanical properties. It is also known that bone tissue in general must be modelled as a Cosserat or second gradient material (Buechner, Lakes, 2003; Madeo et al., 2013) and so it can be regarded as a metamaterial even before reconstruction with grafted metamaterials.

For instance, the initial discontinuity surface, between the existing bone tissue and the bio-resorbable material used as a scaffold for tissue growth, could persist at the end of the remodelling process. This interface could be modelled with material properties, the techniques in dell'Isola, Romano (1986, 1987a,b) and dell'Isola, Kosinski (1993) are a possibility, and greatly influences the transmission and reflection of mechanical waves: it is therefore possible to devise methods to determine the properties of the metamaterial resulting at the end of the complex remodelling process; this metamaterial can be described by using the phenomena in Placidi et al. (2013) or in Madeo et al. (2013), where in the latter the effects of fluid flow in a deformable porous matrix are taken into account (it seems that bone interstitial fluids play an important role in bone growth processes, see section 5.5).

\subsection{Optimal design techniques for bone reconstruction}

Present models are insufficient to describe the bone remodelling and it is really challenging to add all relevant features necessary to attain a reasonably detailed characterisation of these phenomena.

The difficulty resides in weighing the relative importance of factors whose role in these phenomena is just qualitatively known; efforts, in this direction and for specific situations, are described e.g. in Andreaus, Colloca (2009).

Indeed, it is clear that:

- in reconstructed bones, the tissue may be constituted by a mixture of living bone and resorbable biomaterial, the latter used as a scaffold for the tissue growth;

- both bone and bio-resorbable material are porous with assorted sizes of pores and have a solid matrix with different hierarchies of structures; thus it is evident that micro-structured or higher gradient continuum theories are needed (Eringen, 2002; Green, Rivlin, 1964a,b,c, 1965; dell'Isola, Seppecher, 1995; dell'Isola, Sciarra, Vidoli, 2009; dell'Isola, Seppecher, Madeo, 2012); 
- porosity and average density of the porous matrix can be regarded as independent variables, thus requiring the introduction of a micro-structured continuum theory;

- the interstitial fluid is filling the different networks of interconnected or partially disconnected pores and its mechanical behaviour may widely vary in different conditions, showing capillary and viscosity effects;

- a bio-resorbable material can be a metamaterial designed to favour its final total resorption: actually, its property determination is part of the problem to be solved;

- both the living tissue and the bio-resorbable material can be regarded as composite materials with reinforcement fibres (Cowin, 1983, 2008);

- there are probably different mechanisms controlling the resorption of bone and bio-resorbable material and the synthesis of the bone tissue: therefore it may become necessary to introduce more complicated evolution equations for the stimulus or maybe even more stimulus fields.

So, a set of integro-differential equations is to be introduced, possibly with timedelay effects, which takes into account the aspects describing the global growth and remodelling phenomena.

Moreover, the clinical requirements are clear:

- the reconstructed bone needs to resemble the natural bone as close as possible; therefore in principle, the bio-resorbable material should be completely resorbed at the end of the process and, during the resorptionremodelling process, cavities should be avoided since they prevent the formation of new bone tissue;

- remodelling and reformation process should last a reasonable period of time and the mechanical stability of the system must be continuously assured.

At this stage, an optimal problem for bone implant design may be knowingly formulated. It consists in i) defining the characteristics, like initial mass and geometrical positioning of the bio-resorbable material for bone reconstruction, which satisfy the previously listed conditions and ii) minimising the time necessary to reach the target bio-mechanical properties of the remodelled bone. A similar problem is addressed in Andreaus et al. (2011).

A multidisciplinary research group is required for the necessary skills: the numerical modelling demands the formulation of non-standard multiphysics codes, possibly with free-moving boundary problems, involving evolution of a sharp separation between regions with different properties (dell'Isola, Romano, 1987a,b; Eremeyev, Freidin, Sharipova, 2003), in which the constitutive equations are part of the unknown fields to be determined.

\subsection{Modelling of capillary properties in biological tissue with narrow channels.}

Mechanosensing and the related stimulus for tissue growth seem to be influenced by interstitial fluid flow in the narrow channel network present in many biological 
tissues (Madeo, Lekszycki, dell'Isola, 2011; Lekszycki, dell'Isola, 2012; Madeo et al., 2012; Forest, Cordero, Busso, 2011). The modalities of this flow depend on the fluid characteristics and capillary properties of the fluid-channel interface.

In the authors' opinion it is therefore very important to add to the models presented in Federico, Herzog (2008a,c) and Federico, Grillo (2012) the features necessary for a correct description of these phenomena (Seppecher, 1996a,b, 2002).

For living tissues, the results in Madeo, dell'Isola, Darve (2013) may be extended to introduce the concept of interfacial energy density in a REV: the target is a macroscopic model which takes into account wettability of microchannels and density of transported solutes, yet in a simplified way.

Moreover, special attention should be paid to the role of Brinkman dissipation (dell'Isola, Madeo, Seppecher, 2009; Giusteri, Marzocchi, Musesti, 2011; Giusteri, 2013) in the mechanically driven stimulus for the growth of living tissues. Although the phenomena involved in the mechanosensing are not yet completely understood, the influence of higher gradients of velocity field is widely asserted.

Finally, to describe the evolution of the stimulus, it is clearly convenient to adopt equations for which optimised numerical codes are already available: the integral operators up-to-now introduced to calculate the stimulus (cited in section 4.2) have implied considerable difficulties especially with regard to the computational burden.

\section{Conclusions}

Classical field theories are considered nowadays as a nearly sterile research activity. Instead -in the authors' opinion- the most recent developments, not only those in the very limited part of the literature explored here, prove that field theories still are fertile tools to solve many scientific problems and to put into effect promising technological applications.

The assumptions, which usually are considered to be immovable paradigms of continuum mechanics, conversely seem to have a much more restricted applicability and their removal open unexpected scientific possibilities towards technological innovations. Many new phenomena, with very interesting possibilities of applications, simply wait to be observed and understood.

A remark is here pertinent: the theories of continua with micro-structures are indeed very old, as it seems they were formulated by Piola in a pristine form. However, they were not extensively used in the applications: this circumstance has erroneously been interpreted as a sign of their lack of efficacy or their restricted applicability.

On the contrary, in the opinion of the authors, they were not used simply because the exercises and problems formulated in their framework have presented up to now insurmountable technical difficulties.

Nowadays the tools of numerical analysis, coupled with very versatile software packages and powerful computers, allow for the easiest implementation of non-standard mathematical models, as never experienced before.

This is the historical moment where non-standard continuum models should find their fastest development. 
Acknowledgement The research project presented in this paper has been partially supported by the International Research Centre for the Mathematics and Mechanics of Complex Systems "M\&MoCS" of the Università de L'Aquila. The authors thank Francesco dell'Isola and Angelo Luongo for interesting discussions on the topics presented here, and Luca Placidi for his friendly criticism.

\section{References}

Alessandroni, S., Andreaus, U., dell'Isola, F., Porfiri M. (2005). A passive electric controller for multimodal vibrations of thin plates. Computers and Structures, 83, 1236-1250.

Alibert, J.J., Seppecher, P., dell'Isola, F. (2003). Truss modular beams with deformation energy depending on higher displacement gradients. Mathematics and Mechanics of Solids, 8, 51-73.

Altenbach, H., Eremeyev, V.A. (2008). Analysis of the viscoelastic behavior of plates made of functionally graded materials. ZAMM Zeitschrift für Angewandte Mathematik und Mechanik, 88(5), 332-341.

Altenbach, H., Eremeyev, V.A., Morozov, N.F. (2009). Linear theory of shells taking into account surface stresses. Doklady Physics, 54(12), 531-535.

Altenbach, H., Eremeyev, V.A. (2009). Eigen-vibrations of plates made of functionally graded material. Computers, Materials and Continua, 9(2), 153-177.

Altenbach, H., Eremeyev, V.A. (2009). On the bending of viscoelastic plates made of polymer foams. Acta Mechanica, 204(3-4), 137-154.

Altenbach, H., Eremeyev, V.A. (2009). On the linear theory of micropolar plates. ZAMM Zeitschrift für Angewandte Mathematik und Mechanik, 89(4), 242256 .

Altenbach, H., Eremeyev, V.A. (2010). On the effective stiffness of plates made of hyperelastic materials with initial stresses. International Journal of NonLinear Mechanics, 45(10), 976-981.

Altenbach, H., Eremeyev, V.A., Lebedev, L.P., Rendón, L.A. (2010). Acceleration waves and ellipticity in thermoelastic micropolar media, Archive of Applied Mechanics, 80(3), 217-227.

Altenbach, H., Eremeyev, V.A., Morozov, N.F. (2010). On equations of the linear theory of shells with surface stresses taken into account. Mechanics of Solids, 45(3), 331-342.

Altenbach, H., Eremeyev, V.A., Lebedev, L.P. (2010). On the existence of solution in the linear elasticity with surface stresses. ZAMM Zeitschrift für Angewandte Mathematik und Mechanik, 90(3), 231-240.

Altenbach, H., Eremeyev, V.A., Lebedev, L.P. (2011). On the spectrum and stiffness of an elastic body with surface stresses. ZAMM Zeitschrift für Angewandte Mathematik und Mechanik, 91(9), 699-710. 
Altenbach, H., Eremeyev, V.A. (2011). On the shell theory on the nanoscale with surface stresses. International Journal of Engineering Science, 49(12), 1294-1301.

Altenbach, H., Eremeyev, V.A., Morozov, N.F. (2012). Surface viscoelasticity and effective properties of thin-walled structures at the nanoscale. International Journal of Engineering Science, 59 83-89.

Andreaus, U., Giorgio, I., Lekszycki, T. (2013). A 2-D continuum model of a mixture of bone tissue and bio-resorbable material for simulating mass density redistribution under load slowly variable in time. ZAMM Zeitschrift für Angewandte Mathematik und Mechanik, DOI:10.1002/zamm.201200182. Published on-line.

Andreaus, U., dell'Isola, F., Porfiri, M. (2004). Piezoelectric passive distributed controllers for beam flexural vibrations. Journal of Vibration and Control, $10(5), 625-659$.

Andreaus, U., Colloca, M. (2009). Prediction of micromotion initiation of an implanted femur under physiological loads and constraints using the finite element method. Proceedings of the Institution of Mechanical Engineers Part H, Journal of Engineering in Medicine, 223(5), 589-605.

Andreaus, U., Ruta, G.C. (1998). A review of the problem of the shear centre(s). Continuum Mechanics and Thermodynamics, 10(6), 369-380.

Andreaus, U., Baragatti, P. (2009). Fatigue crack growth, free vibrations, and breathing crack detection of aluminium alloy and steel beams. Journal of Strain Analysis for Engineering Design, 44(7), 595-608.

Andreaus, U., Colloca, M., Iacoviello, D., Pignataro, M. (2011). Optimal-tuning PID control of adaptive materials for structural efficiency. Structural and Multidisciplinary Optimization, 43(1), 43-59.

Andreaus, U., Placidi, L., Rega, G. (2010). Numerical simulation of the soft contact dynamics of an impacting bilinear oscillator. Communications in Nonlinear Science and Numerical Simulation, 15(9), 2603-2616.

Auffray, N., Bouchet, R., Brechet, Y. (2009). Derivation of anisotropic matrix for bi-dimensional strain-gradient elasticity behaviour. International Journal of Solids and Structures, 46(2), 440-454.

Auffray, N., Bouchet, R., Brechet, Y. (2010). Strain gradient elastic homogenization of bidimensional cellular media. International Journal of Solids and Structures, 47(13), 1698-1710.

Auffray, N., dell'Isola, F., Eremeyev, V.A., Madeo, A., Rosi, G. (2013). Analytical continuum mechanics à la Hamilton-Piola: least action principle for second gradient continua and capillary fluids. Mathematics and Mechanics of Solids, DOI:10.1177/1081286513497616. Published on-line.

Barnes., H. A. (1997). Thixotropy - a review. Journal of Non-Newtonian Fluid Mechanics, 70(1-2), 1-33. http://dx.doi.org/10.1016/S0377-0257(97)00004-9. 
Bedford, A. (1985). Hamilton's principle in continuum mechanics. Research notes in mathematics 139, Pitman Advanced Publishing Program.

Belyakov, A.O., Seyranian, A.P., Luongo, A. (2009). Dynamics of the pendulum with periodically varying length. Physica D: Nonlinear Phenomena, 238(16), 1589-1597.

Bellini, C., Di Martino, E. S., Federico, S. (2013). Mechanical Behaviour of the Human Atria, Ann. Biomed. Eng., 41 1478-1490.

Berezovski, A., Maugin, G. A. (2005)a. Stress-induced phase-transition front propagation in thermoelastic solids. European Journal of Mechanics-A/Solids, $24(1), 1-21$

Berezovski, A., Maugin, G.A. (2005)b. On the velocity of a moving phase boundary in solids. Acta mechanica, 179(3), 187-196.

Berezovski, A., Engelbrecht, J., Peets, T. (2010). Multiscale modeling of microstructured solids. Mechanics Research Communications, 37(6), 531-534.

Berezovski, A ., Engelbrecht, J., Berezovski, M. (2011). Waves in microstructured solids: a unified viewpoint of modeling. Acta mechanica, 220(1), 349363.

Berezovski, A., Engelbrecht, J., Maugin, G.A. (2011). Thermoelasticity with dual internal variables. Journal of Thermal Stresses, 34(5-6), 413-430.

Bîrsan, M., Altenbach, H., Sadowski, T., Eremeyev, V.A., Pietras, D. (2012). Deformation analysis of functionally graded beams by the direct approach. Composites Part B: Engineering, 43(3), 1315-1328.

Bîrsan, M., Neff, P. (2013). On the characterization of drilling rotation in the 6-parameter resultant shell theory. In: Pietraszkiewiecz, W. (Ed.), ShellStructures: Theory and Applications., 3, Taylor and Francis Group, London.

Bleustein, J.L. (1967). A note on the boundary conditions of Toupin's strain gradient-theory. Int. J. Solids Structures, 3 1053-1057.

Buechner, P.M., Lakes, R.S. (2003). Size effects in the elasticity and viscoelasticity of bone. Biomechan. Model. Mechanobiol., 1, 295-301.

Camar-Eddine, M., Seppecher, P. (2002) Closure of the set of diffusion functionals with respect to the mosco-convergence. Mathematical Models and Methods in Applied Sciences, 12(8), 1153-1176.

Camar-Eddine, M., Seppecher, P. (2003). Determination of the closure of the set of elasticity functionals. Arch. Rat. Mech. Anal., 170(3), 211-245.

Carcaterra, A., Sestieri, A. (1995). Energy Density Equations and Power Flow in Structures. Journal of Sound and Vibration, 188, 269-282.

Carcaterra, A., Ciappi, E., Iafrati, A., Campana, E.F. (2000). Shock spectral analysis of elastic systems impacting on the water surface. Journal of Sound and Vibration, 229, 579-605. 
Carcaterra, A. (2005). Ensemble energy average and energy flow relationships for nonstationary vibrating systems. Journal of Sound and Vibration, 288, 751-790.

Carcaterra, A., Akay, A., Ko, I.M. (2006). Near-irreversibility in a conservative linear structure with singularity points in its modal density. Journal of the Acoustical Society of America, 119, 2141-2149.

Carcaterra, A., Akay, A. (2007). Theoretical foundations of apparent-damping phenomena and nearly irreversible energy exchange in linear conservative systems. Journal of the Acoustical Society of America, 12, 1971-1982.

Carcaterra, A., Akay, A. (2011). Dissipation in a finite-size bath. Physical Review E, 84(1).

Contrafatto, L., Cuomo, M. (2002). A new thermodynamically consistent continuum model for hardening plasticity coupled with damage. International Journal of Solids and Structures, 39, 6241-6271.

Ciancio, D., Carol, I., Cuomo, M. (2007). Crack opening conditions at 'corner nodes' in FE analysis with cracking along mesh lines. Engineering Fracture Mechanics, 74(13), 1963-1982.

Ciancio, D., Carol, I., Cuomo, M. (2006). On inter-element forces in the FEMdisplacement formulation, and implications for stress recovery. International Journal for Numerical Methods in Engineering, 66(3), 502-528.

Contrafatto, L., Cuomo, M. (2006). A framework of elastic-plastic damaging model for concrete under multiaxial stress states. International Journal of Plasticity 22, 2272-2300.

Contrafatto, L., Cuomo, M. (2005). A globally convergent numerical algorithm for damaging elasto-plasticity based on the Multiplier method. International Journal for Numerical Methods in Engineering, 63, 1089-1125.

Cosserat, E., Cosserat, F. Théorie de Corps déformables. Librairie Scientifique A, Hermann et fils, Paris 1909.

Cowin, S.C. (1983). The mechanical and stress adaptive properties of bone. Annals of Biomedical Engineering, 11, 263-295.

Cowin, S.C. (2008). The estimated elastic constants for a single bone osteonal lamella. Biomechanics and Modeling in Mechanobiology, 7, 1-11.

Christensen, J., de Abajo, F. (2012). Anisotropic Metamaterials for Full Control of Acoustic Waves. Physical Review Letters, 108(12).

Culla, A., Sestieri, A., Carcaterra, A. (2003). Energy flow uncertainties in vibrating systems: Definition of a statistical confidence factor. Mechanical Systems and Signal Processing, 17, 635-663.

Cuomo, M., Ventura, G. (1998). Complementary Energy Approach to Contact Problems Based on Consistent Augmented Lagrangian regularization. Mathematical and Computer Modelling, 28, 185-204. 
Cuomo, M., Contrafatto, L. (2000). Stress rate formulation for elastoplastic models with internal variables based on augmented Lagrangian regularisation. International Journal of Solids and Structures, 37, 3935-3964.

Daher, N., Maugin, G.A. (1986). The method of virtual power in continuum mechanics, Application to media presenting singular surfaces and interfaces. Acta Mechanica, 60, 217-240.

dell'Isola, F., Woźniak, C. (1997)a. On phase transition layers in certain microdamaged two-phase solids. International Journal of Fracture, 83(2), 175-189.

dell'Isola, F., Woźniak, C. (1997)b. On continuum modelling the interphase layers in certain two-phase elastic solids. ZAMM Zeitschrift für Angewandte Mathematik und Mechanik, 77(7), 519-526.

dell'Isola, F., Romano, A. (1986). On a general balance law for continua with an interface. Ricerche di Matematica, 35, 325-337.

dell'Isola, F., Romano, A. (1987)a. On the derivation of thermomechanical balance equations for continuous systems with a nonmaterial interface. International Journal of Engineering Science, 25, 1459-1468.

dell'Isola, F., Romano, A. (1987)b. A phenomenological approach to phase transition in classical field theory. International Journal of Engineering Science, 25, 1469-1475.

dell'Isola, F., Kosinski, W. (1993). Deduction of thermodynamic balance laws for bidimensional nonmaterial directed continua modelling interphase layers. Archives of Mechanics, 45, 333-359.

dell'Isola, F. Gouin, H., Seppecher, P. (1995). Radius and surface tension of microscopic bubbles by second gradient theory. Comptes rendus de l'Académie des Sciences Série IIb, 320, 211-216.

dell'Isola, F. Seppecher, P. (1995). The relationship between edge contact forces, double force and interstitial working allowed by the principle of virtual power. Comptes rendus de l'Académie des Sciences Série IIb, 321, 303-308.

dell'Isola, F., Rosa, L., Woźniak, C. (1997). Dynamics of solids with micro periodic nonconnected fluid inclusions. Archive of Applied Mechanics, 67(4), $215-228$.

dell'Isola, F., Hutter, K. (1998). What are the dominant thermomechanical processes in the basal sediment layer of large ice sheets? Proceedings of the Royal Society of London. Series A: Mathematical, Physical and Engineering Sciences, 454, 1169-1195.

dell'Isola, F., Rosa, L., Woźniak, C. (1998). A micro-structured continuum modelling compacting fluid-saturated grounds: The effects of pore-size scale parameter. Acta Mechanica, 127(1-4), 165-182.

dell'Isola, F., Vidoli, S. (1998)a. Damping of bending waves in truss beams by electrical transmission lines with PZT actuators. Archive of Applied Mechanics, 68(9), 626-636. 
dell'Isola, F., Vidoli, S. (1998)b. Continuum modelling of piezoelectromechanical truss beams: An application to vibration damping. Archive of Applied Mechanics, 68(1), 1-19.

dell'Isola, F., Guarascio, M. Hutter, K.A. (2000). Variational approach for the deformation of a saturated porous solid. A second-gradient theory extending Terzaghi's effective stress principle. Archive of Applied Mechanics, 70, 323337.

dell'Isola, F., Madeo, A., Placidi, L. (2012). Linear plane wave propagation and normal transmission and reflection at discontinuity surfaces in second gradient 3D Continua. ZAMM - Zeitschrift für Angewandte Mathematik und Mechanik, 92(1), 52-71.

dell'Isola, F., Sciarra, G., Vidoli, S. (2009). Generalized Hooke's law for isotropic second gradient materials. Proceedings of the Royal Society of London. Series A: Mathematical, Physical and Engineering Sciences, 465, 2177-2196.

dell'Isola, F., Madeo, A., Seppecher, P. (2009). Boundary conditions at fluidpermeable interfaces in porous media: A variational approach. International Journal of Solids and Structures, 46(17), 3150-3164.

dell'Isola, F., Seppecher, P., Madeo, A. (2012). How contact interactions may depend on the shape of Cauchy cuts in N-th gradient continua: approach à la D'Alembert. ZAMP - Zeitschrift für Angewandte Mathematik und Physik, $63,1119-1141$.

dell'Isola, F., Placidi, L. (2012). Variational principles are a powerful tool also for formulating field theories. Variational Models and Methods in Solid and Fluid Mechanics CISM Courses and Lectures, 535, 1-15.

Del Vescovo, D., Fregolent, A. (2005). Assessment of fresco detachments through a non-invasive acoustic method. Journal of Sound and Vibration, 284, 10151031.

Del Vescovo, D., Fregolent, A. (2009). Theoretical and experimental dynamic analysis aimed at the improvement of an acoustic method for fresco detachment diagnosis. Mechanical Systems and Signal Processing, 23(7), 2312-2319, doi:10.1016/j.ymssp.2009.02.009.

Di Egidio, A., Luongo, A., Paolone, A. (2007). Linear and non-linear interactions between static and dynamic bifurcations of damped planar beams. International Journal of Non-Linear Mechanics, 42(1), 88-98.

Engheta, N., Ziolkowski, R. W. (2006). Metamaterials: Physics and Engineering Explorations, Wiley 83 Sons.

Epstein, M., Segev, R. (1980). Differentiable manifolds and the principle of virtual workin continuum mechanics. Journal of Mathematical Physics, 21(5), $1243-1245$.

Epstein, M. (2010). The geometrical language of continuum mechanics, Cambridge. 
Eremeyev, V.A., Freidin, A.B., Sharipova, L.L. (2003). Nonuniqueness and Stability in Problems of Equilibrium of Elastic Two-Phase Bodies. Doklady Physics, 48(7), 359-363.

Eremeyev, V.A., Pietraszkiewicz, W. (2004). The nonlinear theory of elastic shells with phase transitions. Journal of Elasticity, 74(1), 67-86.

Eremeyev, V.A. (2005). Acceleration waves in micropolar elastic media. Doklady Physics, 50(4), 204-206.

Eremeyev, V.A., Pietraszkiewicz, W. (2006). Local symmetry group in the general theory of elastic shells. Journal of Elasticity, 85(2), 125-152.

Eremeyev, V.A., Zubov, L.M. (2007). On constitutive inequalities in nonlinear theory of elastic shells. ZAMM - Zeitschrift für Angewandte Mathematik und Mechanik, 87(2), 94-101.

Eremeyev, V.A., Altenbach, H., Morozov, N.F. (2009). The influence of surface tension on the effective stiffness of nanosize plates. Doklady Physics, 54(2), $98-100$.

Eremeyev, V.A., Lebedev, L.P. (2013). Existence of weak solutions in elasticity. Mathematics and Mechanics of Solids, 18, 204-217.

Eremeyev, V.A., Pietraszkiewicz, W. (2011). Thermomechanics of shells undergoing phase transition. Journal of the Mechanics and Physics of Solids, 59, $1395-1412$.

Eringen, A.C., Suhubi, E.S. (1964)a. Nonlinear theory of simple microelastic solids: I. Int. J. Eng. Sci., 2, 189-203.

Eringen, A.C., Suhubi, E.S. (1964)b. Nonlinear theory of simple microelastic solids: II. Int. J. Eng. Sci., 2, 389-404.

Eringen, A.C., Edelen, D.G.B. (1972). On nonlocal Elasticity. Int. J. Eng. Sci., $10,233-248$.

Eringen, A. C. (1999). Microcontinuum Field Theories I. Foundations and Solids. Springer Verlag.

Eringen, A.C. (2001). Microcontinuum Field Theories, Springer-Verlag, New York.

Eringen, A.C. (2002). Nonlocal continuum field theories, Springer.

Erofeyev, V.I., Potapov, A.I. (1993). Longitudinal Strain Waves in NonLinearly-Elastic Media with Couple Stresses. Int. J. Non-Linear Mechanics, 28(4), 483-488.

Erofeyev, V.I., Pavlov, I.S. Leontiev, N.V. (2013). A Mathematical Model for Investigation of Nonlinear Wave Processes in a 2D Granular Medium Consisting of Spherical Particles. Composites: Mechanics, Computations, Applications, An Int. J., 4(3), 239-255. 
Federico, S., Grillo, A., Herzog, W. (2004). A transversely isotropic composite with a statistical distribution of spheroidal inclusions: A geometrical approach to overall properties. Journal of the Mechanics and Physics of Solids, 52(10), 2309-2327.

Federico, S., Grillo, A., La Rosa, G., Giaquinta, G., Herzog, W. (2005). A Transversely Isotropic, Transversely Homogeneous Microstructural-Statistical Model of Articular Cartilage. JBIOM, 38, 2008-2018.

Federico, S., Herzog, W. (2008)a. On the permeability of fibre-reinforced porous materials. International Journal of Solids and Structures, 45(7-8), 2160-2172.

Federico, S., Herzog, W. (2008)b. Towards an analytical model of soft biological tissues. Journal of Biomechanics, 41(16), 3309-3313.

Federico, S., Herzog, W. (2008)c. On the Anisotropy and Inhomogeneity of Permeability in Articular Cartilage. Biomech. Model. Mechanobiol, 7, 367378.

Federico, S., Gasser, T.C. (2010). Nonlinear elasticity of biological tissues with statistical fibre orientation. Journal of the Royal Society Interface, 7(47), 955966.

Federico, S., Grillo, A. (2012). Elasticity and Permeability of Porous FibreReinforced Materials Under Large Deformations. Mech. Mat., 44, 58-71.

Ferretti, M., Madeo, A., dell'Isola, F., Boisse, P. (2013). Modelling the onset of shear boundary layers in fibrous composite reinforcements by second gradient theory. ZAMP - Zeitschrift für angewandte Mathematik und Physik. Published On-Line.

Forest, S., Sievert, R. (2006). Nonlinear microstrain theories. Int. J. Solids Struct., 43, 7224-7245.

Forest, S. (2009). Micromorphic Approach for Gradient Elasticity, Viscoplasticity, and Damage. Journal of Engineering Mechanics, 135(3), 117-131.

Forest, S., Cordero, N.M., Busso, E.P. (2011). First vs. second gradient of strain theory for capillarity effects in an elastic fluid at small length scales. Computational Materials Science, 50, 1299-1304.

Gatignol, R., Seppecher, P. (1986). Modelisation of fluid-fluid interfaces with material properties. Journal de Mécanique Théorique et Appliquée, 225-247.

Germain, P. (1973)a. La méthode des puissances virtuelles en mécanique des milieux continus. Premiére partie. Théorie du second gradient. Journal de Mécanique, 12, 235-274.

Germain, P. (1973)b. The method of virtual power in continuum mechanics. Part 2: Microstructure. SIAM, Journal of Applied Mathematics, 25, 556-575.

Ghiba, I.D., Neff, P., Madeo, A., Placidi, L., Rosi, G. (2013). The relaxed linear micromorphic continuum: existence, uniqueness and continuous dependence in dynamics. Submitted to Mathematics and Mechanics of Solids. 
Giorgio, I., Culla, A., Del Vescovo, D. (2009). Multimode vibration control using several piezoelectric transducers shunted with a multiterminal network. Archive of Applied Mechanics, 79(9), 859-879.

Giusteri, G. G., Marzocchi, A., Musesti, A. (2011). Nonsimple isotropic incompressible linear fluids surrounding one-dimensional structures. Acta Mechanica, 217(3-4), 191-204, doi:10.1007/s00707-010-0387-5.

Giusteri, G. G. (2013). The multiple nature of concentrated interactions in second-gradient dissipative liquids. ZAMP - Zeitschrift für Angewandte Mathematik und Physik, 64(2), 371-380, doi:10.1007/s00033-012-0229-5.

Green, A.E., Rivlin, R.S. (1964)a. Multipolar continuum mechanics. Archive for Rational Mechanics and Analysis, 17(2), 113-114.

Green, A.E., Rivlin, R.S. (1964)b. Simple force and stress multipoles. Archive for Rational Mechanics and Analysis, 16, 325-353.

Green, A.E., Rivlin, R.S. (1964)c. On Cauchy's equations of motion. ZAMP Zeitschrift für Angewandte Mathematik und Physik 15, 290-292.

Green, A.E., Rivlin, R.S. (1965). Multipolar continuum mechanics: functional theory I. Proceedings of the Royal Society of London. Series A: Mathematical, Physical and Engineering Sciences, 284, 303-324.

Haseganu, E.M., Steigmann, D.J. (1996). Equilibrium analysis of finitely deformed elastic networks. Computational mechanics, 17, 359-373.

Hellinger, E. (1913). Die allgemeinen Ansätze der Mechanik der Kontinua, Encyklopädie der mathematischen Wissenschaften. Bd. IV-4, Hft. 5.

Jamal, M., Lahlou, L., Midani, M., Zarouni, H., Limam, A., Damil, N., PotierFerry, M. (2003). A semi-analytical buckling analysis of imperfect cylindrical shells under axial compression. International Journal of Solids and Structures, 40(5), 1311-1327.

Klawonn, A., Neff, P., Rheinbach, O., Vanis, S. (2011). FETI-DP domain decomposition methods for elasticity with structural changes: P-elasticity. ESAIM: Math. Mod. Num. Anal., 45, 563-602.

Kolpakovs, A.G. (1985). Determination of the average characteristics of elastic frameworks. Journal of Applied Mathematics and Mechanics, 49(6), 739-745. (Elsevier http://www.sciencedirect.com/science/article/pii/0021892885900115).

Kroner, E. (1968). Mechanics of Generalized Continua. Springer.

Lagrange, J.L. (1788). Mécanique Analytique, Editions Jaques Gabay, Sceaux.

Landau, L.D., Lifshitz, E.M. (1975). The Classical Theory of Fields 2, 4th ed., Butterworth-Heinemann.

Landau, L.D., Lifshitz, E.M. (1976). Mechanics 1, 3rd ed., Butterworth-Heinemann. 
Landau, L.D., Lifshitz, E.M. (1977). Quantum Mechanics: Non-Relativistic Theory 3, 3rd ed., Pergamon Press.

Lebedev, L.P., Cloud, M.J., Eremeyev, V.A. (2010). Tensor Analysis with Applications in Mechanics. New Jersey: World Scientific.

Lekszycki, T., dell'Isola, F. (2012). A mixture model with evolving mass densities for describing synthesis and resorption phenomena in bones reconstructed with bio-resorbable materials. ZAMM - Journal of Applied Mathematics and Mechanics / Zeitschrift für Angewandte Mathematik und Mechanik, 92(6), 426-444.

Lee, S. H., Park, C. M., Seo, Y. M., Wang, Z. G., Kim, C. K. (2010). Composite Acoustic Medium with Simultaneously Negative Density and Modulus. Physical Review Letters, 104(5), doi:10.1103/PhysRevLett.104.054301.

Lehoucq, R.B., Silling, S.A. (2008). Force flux and the peridynamic stress tensor. Journal of the Mechanics and Physics of Solids, 56(4), 1566-1577.

Limam, A., Lee, L.-H., Corona, E., Kyriakides, S. (2010). Inelastic wrinkling and collapse of tubes under combined bending and internal pressure. International Journal of Mechanical Sciences, 52(5), 637-647.

Luongo, A., Rega, G., Vestroni, F. (1986). On nonlinear dynamics of planar shear indeformable beams. Journal of Applied Mechanics, Transactions ASME, 53(3), 619-624.

Luongo, A. (2001). Mode localization in dynamics and buckling of linear imperfect continuous structures. Nonlinear Dynamics, 25(1-3), 133-156.

Luongo, A., Paolone, A., Di Egidio, A. (2003). Multiple Timescales Analysis for 1:2 and 1:3 Resonant Hopf Bifurcations. Nonlinear Dynamics, 34(3-4), 269-291.

Luongo, A., Di Egidio, A. (2005). Bifurcation equations through multiple-scales analysis for a continuous model of a planar beam. Nonlinear Dynamics, 41, $171-190$

Luongo, A., Romeo, F. (2006). A Transfer-matrix-perturbation approach to the dynamics of chains of nonlinear sliding beams. Journal of Vibration and Acoustics, 128, 190-196.

Luongo, A., Zulli, D., Piccardo, G. (2009). On the effect of twist angle on nonlinear galloping of suspended cables. Computers $\&$ Structures, 87, 10031014 .

Madeo, A., Gavrilyuk, S. (2010). Propagation of acoustic waves in porous media and their reflection and transmission at a pure-fluid/porous-medium permeable interface European. Journal of Mechanics-A/Solids, 29(5), 897-910.

Madeo, A., Lekszycki, T., dell'Isola, F. (2011). A continuum model for the biomechanical interactions between living tissue and bio-resorbable graft after bone reconstructive surgery. Comptes Rendus - Mecanique, 339(10), 625-640. 
Madeo, A., George, D., Lekszycki, T., Nierenberger, M., Rémond, Y. (2012). A second gradient continuum model accounting for some effects of microstructure on reconstructed bone remodelling. Comptes Rendus Mécanique, $340(8), 575-589$.

Madeo, A., Djeran-Maigre, I., Rosi, G., Silvani, C. (2013). The effect of fluid streams in porous media on acoustic compression wave propagation, transmission, and reflection. Continuum Mechanics and Thermodynamics, 25, 173 196 .

Madeo, A., dell'Isola, F., Darve, F. (2013). A continuum model for deformable, second gradient porous media partially saturated with compressible fluids. Journal of the Mechanics and Physics of Solids, 61, 2196-2211.

Manktelow, K., Leamy, M.J., Ruzzene, M. (2013)a. Topology Design and Optimization of Nonlinear Periodic Materials. Journal of the Mechanics and Physics of Solids, 61(12), 2433-2453.

Manktelow, K., Leamy, M.J., Ruzzene, M. (2013)b. Comparison of Asymptotic and Transfer Matrix Approaches for Evaluating Intensity-Dependent Dispersion in Nonlinear Photonic and Phononic Crystals. Wave Motion, 50, 494-508.

Maugin, G. A., Muschik, W. (1994)a. Thermodynamics with Internal Variables. Part I. General Concepts. Journal of Non-Equilibrium Thermodynamics, 19(3), 217-249.

Maugin, G. A., Muschik, W. (1994)b. Thermodynamics with internal variables. Part II. Applications. Journal of Non-Equilibrium Thermodynamics, 19(3), 250-289.

Maugin, G.A. (2013). The principle of virtual power: from eliminating metaphysical forces to providing an efficient modelling tool In memory of Paul Germain (1920-2009). Cont. Mech. Thermodyn. Published On-Line DOI 10.1007/s00161-011-0196-7, 1-20.

Maugin, G.A., Metrikine, A.V. (eds.) (2010). Mechanics of generalized Continua One hundred years after the Cosserats. Springer.

Maurini, C., dell'Isola, F., Del Vescovo, D. (2004). Comparison of piezoelectronic networks acting as distributed vibration absorbers. Mechanical Systems and Signal Processing, 18, 1243-1271.

Maurini, C., Pouget, J., dell'Isola, F. (2004). On a model of layered piezoelectric beams including transverse stress effect. International Journal of Solids and Structures, 4, 4473-4502.

Maurini, C., Pouget, J., dell'Isola, F. (2006). Extension of the Euler-Bernoulli model of piezoelectric laminates to include 3D effects via a mixed approach. Computers and Structures, 84(22-23), 1438-1458.

McBride, A.T., Javili, A., Steinmann, P. Bargmann, S. (2011). Geometrically nonlinear continuum thermomechanics with surface energies coupled to diffusion. Journal of the Mechanics and Physics of Solids, 59, 2116-2133. 
McBride, A.T., Mergheim, J., Javili, A., Steinmann, P., Bargmann, S. (2012). Micro-to-macro transitions for heterogeneous material layers accounting for in-plane stretch. Journal of the Mechanics and Physics of Solids, 60, 12211239 .

Michel, G., Limam, A., Jullien, J.F. (2000). Buckling of cylindrical shells under static and dynamic shear loading. Engineering Structures, 22(5), 535-543.

Mindlin, R.D. (1964). Micro-structure in linear elasticity. Archive for Rational Mechanics and Analysis, 16, 51-78.

Mindlin, R.D. (1965). Second gradient of strain and surface tension in linear elasticity. International Journal of Solids and Structures, 1, 417-438.

Mindlin, R.D., Eshel, N.N. (1968). On first strain-gradient theories in linear elasticity. International Journal of Solids and Structures, 4, 109-124.

Misra, A., Chang, C.S. (1993). Effective Elastic Moduli of Heterogeneous Granular Solids. International Journal of Solids and Structures, 30, 2547-2566.

Misra, A., Yang, Y. (2010). Micromechanical model for cohesive materials based upon pseudo-granular structure. International Journal of Solids and Structures, 47, 2970-2981.

Misra, A., Singh, V. (2013). Micromechanical model for viscoelastic-materials undergoing damage. Continuum Mechanics and Thermodynamics, 25, 1-16.

Misra, A., Ching, W.Y. (2013). Theoretical nonlinear response of complex single crystal under multi-axial tensile loading. Scientific Reports, 3, 1488.

Mullender, M. G., Huiskes, R. (1995). Proposal for the Regulatory Mechanism of Wolff's Law. Journal of Orthopaedic Research, 13, 503-512.

Münch, I., Wagner, W., Neff,P. (2011). Transversely isotropic material: nonlinear Cosserat versus classical approach. Cont. Mech. Thermod., 23, 27-34.

Nadler, B., Papadopoulos, P., Steigmann, D.J. (2006). Multiscale constitutive modeling and numerical simulation of fabric material. International Journal of Solids and Structures, 43, 206-221.

Narisetti, R.K., Ruzzene, M., Leamy, M.J. (2012). Study of Wave Propagation in Strongly Nonlinear Periodic Lattices Using a Harmonic Balance Approach. Wave Motion, 49, 394-410.

Narisetti, R.K., Ruzzene, M., Leamy, M.J. (2011). A Perturbation Approach for Analyzing Dispersion and Group Velocities in Two-Dimensional Nonlinear Periodic Lattices. Journal of Vibration and Acoustics, 133(6), 1-12.

Neff, P. (2002). On Korn's first inequality with non-constant coefficients. Royal Society of Edinburgh - Proceedings A, 132(1), 221-243.

Neff, P., Wieners, C. (2003). Comparison of models for finite plasticity. A numerical study. Comput. Visual. Sci., 6, 23-35. 
Neff, P. (2004)a. A geometrically exact Cosserat shell-model including size effects, avoiding degeneracy in the thin shell limit. Part I: Formal dimensional reduction for elastic plates and existence of minimizers for positive Cosserat couple modulus. Continuum Mechanics and Thermodynamics, 16(6), 577628.

Neff, P. (2004)b. Geometrically exact Cosserat theory for bulk behaviour and thin structures. Modelling and mathematical analysis. Signatur HS 7/0973, Habilitationsschrift, Universitäts- und Landesbibliothek, Technische Universität Darmstadt, Darmstadt.

Neff, P., Chełmiński, K. (2005). Infinitesimal elastic-plastic Cosserat micropolar theory. Modelling and global existence in the rate independent case. Proc. Roy. Soc. Edinb. A, 135, 1017-1039.

Neff, P.(2006)a. A finite-strain elastic-plastic Cosserat theory for polycrystals with grain rotations. International Journal of Engineering Science, 44(8-9), $574-594$.

Neff, P. (2006)b. The Cosserat couple modulus for continuous solids is zero viz the linearized Cauchy-stress tensor is symmetric. ZAMM - Zeitschrift für Angewandte Mathematik und Mechanik, 86(11), 892-912.

Neff, P. (2006)c. Existence of minimizers for a finite-strain micromorphic elastic solid. Proc. Roy. Soc. Edinb. A, 136, 997-1012.

Neff, P. (2007). A geometrically exact planar cosserat shell-model with microstructure: Existence of minimizers for zero cosserat couple modulus. Mathematical Models and Methods in Applied Sciences, 17(3), 363-392.

Neff, P., Chełmiński, K. (2007). A geometrically exact Cosserat shell model for defective elastic crystals Justification via-convergence. Interfaces and Free Boundaries, 9(4), 455-492.

Neff, P., Forest, S. (2007). A geometrically exact micromorphic model for elastic metallic foams accounting for affine microstructure. Modelling, existence of minimizers, identification of moduli and computational results. J. Elasticity, 87, 239-276.

Neff, P., Münch, I. (2008). Curl bounds Grad on SO(3). ESAIM: Control, Optimisation and Calculus of Variations, 14, 148-159.

Neff, P., Jeong, J. (2009). A new paradigm: The linear isotropic Cosserat model with conformally invariant curvature energy. ZAMM - Zeitschrift für Angewandte Mathematik und Mechanik, 89(2), 107-122.

Neff, P., Jeong, J., Münch, I., Ramezani, H. (2009). Mean field modeling of isotropic random Cauchy elasticity versus microstretch elasticity. Z. Angew. Math. Phys., 3, 479-497.

Neff, P., Jeong, J., Fischle, A. (2010). Stable identification of linear isotropic Cosserat parameters: bounded stiffness in bending and torsion implies conformal invariance of curvature. Acta Mechanica, 211, 237-249. 
Neff, P., Pauly, D., Witsch, K. (2012). Maxwell meets Korn: A new coercive inequality for tensor fields in $R^{N \times N}$ with square inegrable exterior derivative. Math. Meth. Appl. Sci., 35, 65-71.

Neff, P., Ghiba, I.D., Madeo, A., Placidi, L., Rosi, G. (2013) A unifying perspective: the relaxed linear micromorphic continuum. Submitted to Continuum Mechanics and Thermodynamics, http://dx.doi.org/10.1007/s00161013-0322-9.

Neff, P., Pauly, D., Witsch, K. (2013). Poincaré meets Korn via Maxwell: Extending Korn's first inequality to incompatible tensor fields. submitted; http://arxiv.org/abs/1203.2744; Preprint SM-E-753, Universität Duisburg-Essen, Schriftenreihe der Fakultät für Mathematik, http//www.unidue.de/mathematik/preprints.shtml.

Neff, P., Pompe, W. (2013). Counterexamples in the theory of coerciveness for linear elliptic systems related to generalizations of Korn's second inequality. To appear in Z. Angew. Math. Mech, http://arxiv.org/abs/1303.1387, Preprint UDE: SM-UDE-765.

Paolone, A., Vasta, M., Luongo, A. (2006). Flexural-torsional bifurcations of a cantilever beam under potential and circulatory forces I: Non-linear model and stability analysis. International Journal of Non-Linear Mechanics, 41(4), $586-594$

Parks, M.L., Lehoucq, R.B., Plimpton, S.J., Silling, S.A. (2008). Implementing peridynamics within a molecular dynamics code. Comp Phys Comm, 179, $777-783$.

Pideri, C., Seppecher, P. (1997). A second gradient material resulting from the homogenization of an heterogeneous linear elastic medium. Continuum Mechanics and Thermodynamics, 9, 241-257.

Pietraszkiewicz, W., Eremeyev, V.A. (2009). On vectorially parameterized natural strain measures of the non-linear Cosserat continuum. International Journal of Solids and Structures, 46(11-12), 2477-2480.

Pietraszkiewicz, W., Eremeyev, V.A., Konopinska, V. (2007). Extended nonlinear relations of elastic shells undergoing phase transitions. ZAMM - Zeitschrift für Angewandte Mathematik und Mechanik, 87, 150-159.

Pignataro, M., Luongo, A. (1987). Asymmetric interactive buckling of thinwalled columns with initial imperfections. Thin-Walled Structures, 5(5), 365382 .

Piola, G. Memoria intorno alle equazioni fondamentali del movimento di corpi qualsivogliono considerati secondo la naturale loro forma e costituzione, Modena, Tipi del R.D. Camera, 1846 translated by F. dell'Isola, U. Andreaus and L. Placidi in "The complete works of Gabrio Piola: Volume I U. Andreaus, F.dell'Isola, R. Esposito, S. Forest, G.Maier, U. Perego, Editors Springer Verlag. In Preparation (see also www.fdellisola.it). 
Placidi, L., Rosi, G., Giorgio, I., Madeo, A. (2013). Reflection and transmission of plane waves at surfaces carrying material properties and embedded in second-gradient materials. Mathematics and Mechanics of Solids, doi: $10.1177 / 1081286512474016$.

Porfiri, M., dell'Isola, F., Santini, E. (2005). Modeling and design of passive electric networks interconnecting piezoelectric transducers for distributed vibration control. International Journal of Applied Electromagnetics and Mechanics, 21(2), 69-87.

Porubov, A. (2003). Amplification of nonlinear strain waves in solids. World Scientific Publishing Company Incorporated.

Potapov, A.I., Pavlov, I.S., Maugin, G.A. (1999). Nonlinear wave interactions in 1D crystals with complex lattice. Wave Motion, 29(4), 297-312.

Quiligotti, S., Maugin, G.A., dell'Isola, F. (2003). An Eshelbian approach to the nonlinear mechanics of constrained solid-fluid mixtures. Acta Mechanica, 160, 45-60.

Quiligotti, S., Maugin, G. A., dell'Isola, F. (2002). Wave motions in unbounded poroelastic solids infused with compressible fluids. ZAMP - Zeitschrift für angewandte Mathematik und Physik, 53(6), 1110-1138.

Rinaldi, A., Lai, Y.C. (2007)a. Damage Theory Of 2D Disordered Lattices: Energetics And Physical Foundations Of Damage Parameter. Int. J. Plasticity, $23,1796-1825$.

Rinaldi, A., Krajcinovic, D., Mastilovic, S. (2007). Statistical Damage Mechanics and Extreme Value Theory. Int. J. Damage Mech, 16(1), 57-76.

Rinaldi, A., Lai, Y.C. (2007)b. Statistical damage theory of 2D lattices: Energetics and physical foundations of damage parameter. International Journal of Plasticity, 23, 1796-1825.

Rinaldi, A., Krajcinovic, D., Peralta, P., Lai, Y.C. (2008). Lattice models of polycrystalline microstructures: A quantitative approach. Mechanics of $M a-$ terials, 40, 17-36.

Rinaldi, A. (2009). A rational model for 2D Disordered Lattices Under Uniaxial Loading. Int. J. Damage Mech., 18, 233-257.

Rinaldi, A. (2011). Statistical model with two order parameters for ductile and soft fiber bundles in nanoscience and biomaterials. Phys Rev E, 83(4-2).

Rinaldi, A. (2013). Bottom-up modeling of damage in heterogeneous quasibrittle solids. Continuum Mechanics and Thermodynamics, 25(2-4), 359-373.

Rosi, G., Madeo, A., Guyader, J.L. (2013). Switch between fast and slow Biot compression waves induced by "second gradient microstructure" at material discontinuity surfaces in porous media. International Journal of Solids and Structures, 50(10), 1721-1746. 
Rosi, G., Giorgio, I., Eremeyev, V.A. (2013). Propagation of linear compression waves through plane interfacial layers and mass adsorption in second gradient fluids. ZAMM-Journal of Applied Mathematics and Mechanics/Zeitschrift für Angewandte Mathematik und Mechanik, 93(12), 914-927.

Ricci-Curbastro, G., Levi-Civita, T. (1900). Méthodes de calcul différentiel absolu et leurs applications. Mathematische Annalen, 54(1-2), 125-201.

Russo, L. (2004). The Forgotten Revolution: How Science Was Born in 300 BC and Why It Had to Be Reborn. Springer, Berlin.

Sciarra, G., dell'Isola, F., Hutter, K. (2001). A solid-fluid mixture model allowing for solid dilatation under external pressure. Continuum Mechanics and Thermodynamics, 13(5), 287-306.

Sciarra, G., dell'Isola, F., Coussy, O. (2007). Second gradient poromechanics. Int. J. Solids Struct, 44(20), 6607-6629.

Sedov, L.I. (1972). Models of continuous media with internal degrees of freedom. Journal of Applied Mathematics and Mechanics, 32, 803-819.

Sedov, L.I. (1968). Variational Methods of constructing Models of Continuous Media, Irreversible Aspects of Continuum Mechanics and Transfer of Physical Characteristics in Moving Fluids. Springer Vienna, 346-358.

Segev, R. (1986). Forces and the existence of stresses in invariant continuum mechanics. Journal of Mathematical Physics, 27(1), 163-170.

Segev, R. (2000). The geometry of Cauchy's fluxes. Archive for Rational Mechanics and Analysis, 154(3), 183-198.

Seppecher, P. (1987). Etude d'une Modelisation des Zones Capillaires Fluides: Interfaces et Lignes de Contact. Thése de l'Université Paris VI.

Seppecher, P. (1988). Thermodynamique des zones capillaires. Annales de Physique, 13, 13-22.

Seppecher, P. (1989). Etude des conditions aux limites en théorie du second gradient: cas de la capillarité, Comptes rendus de l'Académie des Sciences, 309, 497-502.

Seppecher, P. (1993). Equilibrium of a Cahn and Hilliard fluid on a wall: Influence of the wetting properties of the fluid upon the stability of a thin liquid film. European Journal of mechanics B/fluids, 12, 69-84.

Seppecher, P. (1996)a. A numerical study of a moving contact line in CahnHilliard theory. International Journal of Engineering Science, 34, 977-992.

Seppecher, P. (1996)b. Les Fluides de Cahn-Hilliard. Mémoire d'Habilitation à Diriger des Recherches, Université du Sud Toulon Var.

Seppecher, P. (2001). Line Tension Effect upon Static Wetting. Oil and Gas Science and Technology - Rev. IFP, 56, 77-81.

Seppecher, P. (2002). Second-gradient theory: application to Cahn-Hilliard fluids, in Continuum Thermomechanics. Springer Netherlands, 379-388. 
Steinmann, P. (2008). On Boundary Potential Energies in Deformational and Configurational Mechanics. J. Mech. Phys. Solids, 56, 772-80.

Sunyk, R., Steinmann, P. (2003). On Higher Gradients in Continuum-Atomistic Modelling. Int. J. Solids Structures, 40(24), 6877-6896.

Steigmann, D.J., Ogden, R.W. (1997). Plane deformations of elastic solids with intrinsic boundary elasticity. Proceedings of the Royal Society A: Mathematical, Physical and Engineering Sciences, 453, 853-877.

Steeb, H., Diebels, S. (2004). Modeling thin films applying an extended continuum theory based on a scalar-valued order parameter - Part I: Isothermal case. International Journal of Solids and Structures, 41, 5071-5085.

Steinmann, P., Elizondo, A., Sunyk, R. (2007). Studies of validity of the CauchyBorn rule by direct comparison of continuum and atomistic modelling. Modelling and Simulation in Materials Science and Engineering, 15.

Spivak, M. (1979). A comprehensive introduction to differential geometry, Vol. I and II. Second edition. Publish or Perish, Inc., Wilmington.

Toupin, R.A. (1962). Elastic Materials with couple-stresses. Archive for Rational Mechanics and Analysis, 11, 385-414.

Toupin, R.A. (1964). Theories of elasticity with couple-stress. Archive for Rational Mechanics and Analysis, 17, 85-112.

Yang, Y., Misra, A. (2010). Higher-order stress-strain theory for damage modeling implemented in an element-free Galerkin formulation. Computer Modeling in Engineering and Sciences, 64, 1-36.

Yang, Y., Misra, A. (2012). Micromechanics based second gradient continuum theory for shear band modeling in cohesive granular materials following damage elasticity. International Journal of Solids and Structures, 49, 2500-2514.

Yang, Y., Ching, W.Y., Misra, A. (2011). Higher-order continuum theory applied to fracture simulation of nano-scale intergranular glassy film. Journal of Nanomechanics and Micromechanics, 1, 60-71.

Yeremeyev, V.A., Freidin, A.B., Sharipova, L.L. (2007). The stability of the equilibrium of two-phase elastic solids. Journal of Applied Mathematics and mechanics (PMM), 71, 61-84.

Vidoli, S., dell'Isola, F. (2000). Modal coupling in one-dimensional electromechanical structured continua. Acta Mechanica, 141, 37-50.

Vidoli, S., dell'Isola, F. (2001). Vibration control in plates by uniformly distributed PZT actuators interconnected via electric networks. European Journal of Mechanics, A/Solids, 20(3), 435-456.

Zouhdi, S., Ari, S., Vinogradov, A. P. (2008). Metamaterials and Plasmonics: Fundamentals, Modelling, Applications. Springer-Verlag, New York.

Xu, B., Arias, F., Brittain, S. T., Zhao, X.-M., Grzybowski, B., Torquato, S., Whitesides, G.M. (1999). Making Negative Poisson's Ratio Microstructures by Soft Lithography. Advanced Materials, 11(14), 1186-1189. 
Wolff, J. (1892). Das Gesetz der Transformation der Knochen, (The Law of Bone Remodeling, translated by P. Maquet and R. Furlong, 1986), Springer-Verlag, Berlin. 